(C)2019. This manuscript version is made available under the CC-BY-NC-ND 4.0 license http://creativecommons.org/licenses/by-nc-nd/4.0/

\title{
Toward the Development of a Virtual Spray Test-Rig using the Smoothed Particle Hydrodynamics Method
}

\author{
G. Chaussonnet ${ }^{\mathrm{a}}$, S. Braun ${ }^{\mathrm{a}}$, T. Dauch ${ }^{\mathrm{a}}$, M. Keller ${ }^{\mathrm{a}}$, A. Sänger ${ }^{\mathrm{b}}$, T. Jakobs ${ }^{\mathrm{b}}$, R. Koch ${ }^{\mathrm{a}}$, T. Kolb ${ }^{\mathrm{b}}$, \\ H.-J. Bauera \\ ${ }^{a}$ Insitut für Thermische Strömungsmaschinen, Karlsruher Institut für Technologie (KIT), Karlsruhe, Germany \\ ${ }^{b}$ Insitut für Technische Chemie, Karlsruher Institut für Technologie (KIT), Karlsruhe, Germany
}

\begin{abstract}
In this work we present the numerical simulation of air-assisted liquid atomization at high pressure using the Smoothed Particle Hydrodynamics (SPH) method. Different post-processing tools are applied to facilitate the comparison with experimental observations. This allows to quantitatively validate the numerical method against the experiment, in terms of (i) frequency of the Kelvin-Helmholtz instability that develops on the jet surface, and (ii) statistical distribution of the jet intact length. The qualitative comparison also shows a good prediction of the jet global instability and of the fragmented liquid lumps, with regards to length and time scales. In addition, the post-processing tools also give access to the local parameters of the generated spray in the vicinity of the nozzle, which are not easily accessible in a real experiments. Using these tools, 1D profiles and 2D maps of the liquid phase properties such as the volume fraction, the droplet concentration, the Sauter Mean Diameter (SMD) and the droplet sphericity are presented. Because of the Lagrangian nature of the SPH method, it is also possible to monitor the whole atomization cascade as a causal tree, from the primary instabilities to the spray characteristics. This tree contains various information such as the fragmentation spectrum and the breakup activity, which are of great interest for researchers and engineers. Hence, the capability of the Smoothed Particle Hydrodynamics (SPH) method for simulating air-assisted atomization at high ambient pressure is demonstrated as well as its applicability to realistic configurations. This is a first step towards the development of a complete virtual spray test-rig.
\end{abstract}

Keywords: Smoothed Particle Hydrodynamics, air-assisted breakup, atomization cascade, spray characteristics, virtual spray test-rig

\section{Introduction}

The increase of computational capacity of High Performance Computing (HPC) clusters allows to increase spatial and temporal resolution of numerical simulations. Another possibility of taking advantage of the increasing CPU capacity is to simulate larger geometries for a longer physical time. Both ways of evolution allow for a broader range of resolved time and length scales. In addition, many numerical methods have reached a level of maturity with several validation cases for different physical phenomena. Therefore, it becomes now possible to use numerical simulation as a design tool.

Despite this trend, the design of a spray nozzle based on numerical simulation is still a challenging task. Even though the chain of phenomena transforming a plain liquid jet into a spray is globally known, the details of the mechanisms are not fully understood. Hence, no low-order models can be used to reduce the computational overheads and provide accurate spray characteristics at the same time. In addition, the atomization process involves a wide range of length and time scales. The thickness of a liquid membrane is of the order of one micrometer whereas the diameter of an atomizing nozzle at the industrial scale is in

Email address: geoffroy.chaussonnet@kit.edu (G. Chaussonnet) 
(C)2019. This manuscript version is made available under the CC-BY-NC-ND 4.0 license

http://creativecommons.org/licenses/by-nc-nd/4.0/

the range of centimeters. The breakup time of liquid ligaments is $\sim 100 \mu$ s while converged spray statistics are usually obtained after several seconds. Therefore, the numerical simulation of liquid breakup is still a challenging topic for both researchers and engineers. Experimental investigation on liquid jet atomization is still an active topic, from the onset of the primary instabilities (Matas et al., 2018), to the resulting droplet size distribution and their dependence on the flow (Dhivyaraja et al., 2019). For a comprehensive review on liquid jet atomization, the reader is referred to Dumouchel (2008).

In the last five years, the most relevant works about simulating liquid atomization were achieved by grid based methods, and the spray characteristics could be determined in terms of global Sauter Mean Diameter, or less frequently, in terms of drop size distribution. For instance, Müller et al. (2016) simulated the same configuration as the present study at ambient pressure with compressible Large-Eddy Simulation and the VOF method. They found that the primary instability as well as the breakup frequency were in good agreement with the experiment. Nevertheless they did not investigate the characteristics of the generated spray. In the field of airblast or air-assisted atomization, Shao et al. (2017) investigated the primary breakup in a swirling airblast atomizer with a mass conservative Level Set method, focusing on the primary instabilities only. Warncke et al. (2017) investigated the primary breakup of a planar prefilming airblast atomizer with the VOF method and extracted the drop size distribution. They highlighted the critical dependency of the spray characteristics on the selection of the discriminating threshold of the liquid volume fraction. Li and Soteriou (2018) studied the academical configuration of a jet in cross-flow with a Coupled Level-Set and Volume Of Fluid (CLSVOF) method in a volume of 3x2x3 $\mathrm{cm}^{3}$. They extracted the global SMD for 50 time frames for different density ratios. Salvador et al. (2018) studied the breakup of a liquid fuel jet discharging into a quiescent atmosphere with a Volume Of Fluid (VOF) method in a near-nozzle region of the dimension $2.34 \mathrm{~mm}$. They determined the equivalent drop sizes of the spray from the volume of each liquid structure and extracted the corresponding distribution over a period of $20 \mu \mathrm{s}$. Finally, a new mesh-based method, the Lattice-Botlzmann Method (LBM), has shown promising results in the field of multiphase flows, and especially atomization. In this context, Amirshaghaghi et al. (2018) studied the 2D air-assisted atomization of a liquid sheet with the LBM. However, they only focused their study on the primary instabilities.

As an intermediate conclusion, the determination of the spray characteristics by numerical mesh-based methods based on first principles was achieved in several configurations, but either over a small time period, or in very small volume. The goal of the present work is to demonstrate the advantage of the Smoothed Particle Hydrodynamics (SPH) method to simulate air-assisted atomization at high ambient pressure in industry-oriented nozzles of intermediate size and over a sufficiently long time-span to obtain converged spray characteristics. Furthermore, it will be shown that the use of SPH allows a number of post-processing tools larger that the ones offered by mesh-based methods. The results of the numerical simulation will be compared to experimental observations to validate the present approach. This study is part of the mid-term goal to develop a virtual spray test-rig that could provide a detailed insight of the breakup mechanism both for researchers and engineers.

The SPH method was originally developed in the context of astrophysics (Gingold and Monaghan, 1977) and later adapted to free surface flow (Monaghan and Kocharyan, 1995). Colagrossi and Landrini (2003) and $\mathrm{Hu}$ and Adams (2006) proposed methods to compute interfacial flows. Adami et al. (2010) developed a robust method to compute the surface tension effects. It is is a particle method that is based on a Lagrangian formulation of the fluid using moving interpolation points, the so called particles. These interpolation points carry the physical properties (e.g. mass, volume, momentum and energy). The greatest advantage of the SPH method over grid-based methods in simulating multiphase flow is the advection of the phases interface by the natural arrangement of the particles. Each particle is assigned a phase type (e.g. liquid or gas), and this type remains the same during the entire simulation. Therefore, the overall motion of liquid particles describes the motion of the fluid phase, and the utmost liquid particles determine the free surface of the liquid. Hence, no reconstruction algorithm for the interface is required. The corollary advantage is that large liquid deformations are also handled in a straightforward way, and no local refinement has to be applied, as long as the spatial resolution determined by the particle size is sufficient. Finally, due to the Lagrangian nature of the SPH method, each particle can be individually tracked during the simulation. Hence, trajectories and Lagangian Coherent Structures can be easily built (Sun et al., 2016; Dauch et al., 
(C)2019. This manuscript version is made available under the CC-BY-NC-ND 4.0 license http://creativecommons.org/licenses/by-nc-nd/4.0/

2018). In the particular case of atomization, this unique identification allows to follow each liquid lump during the breakup process. Consequently, the whole atomization cascade (from the liquid jet to the fine spray droplets) can be monitored. From a computational point of view, because of its simple principle, the SPH method has proven superior serial and parallel performance (Braun et al., 2016a).

The SPH method was already applied in the field of atomization. Pereira et al. (2018) studied the internal flow of a pressure atomizer but due to a too large particle size $(500 \mu \mathrm{m})$, they could not resolve the spray characteristics. In the more restrictive field of air-assisted liquid atomization, the application of SPH method was pioneered by Höfler et al. (2011). Braun et al. (2015b) studied a generic 2D planar prefilming airblast atomizer. They demonstrated that the method retrieves the proper ligament structures just prior to the breakup. Dauch et al. (2017) investigated the sector of a realistic annular prefilming airblast atomizer and demonstrated the applicability of SPH to a complex geometry. Finally, Chaussonnet et al. (2018) investigated the same configuration as in the present work, but in a $2 \mathrm{D}$ geometry with different ambient pressure and different type of liquid.

The present paper is organized as follows. The numerical method is presented in Section 2. With regard to applying this method to industrial configurations, the post-processing tools are also described. Then, the reference experiment and its main results are presented in Section 3. The numerical setup of the configuration is described in Section 4. Then the results will be qualitatively discussed in Section 5 and quantitatively in Section 6. The paper ends with a conclusion.

\section{Numerical Model}

\subsection{SPH discretization}

The SPH method is based on the description of any spatial field $f(\boldsymbol{r})$ by a convolution with the Dirac delta function $\delta(\boldsymbol{r})$ :

$$
f(\boldsymbol{r})=\int f\left(\boldsymbol{r}^{\prime}\right) \delta\left(\boldsymbol{r}-\boldsymbol{r}^{\prime}\right) \mathrm{d} \boldsymbol{r}^{\prime}
$$

In order to apply Eq. 1 to discrete particles, the Dirac function is replaced by a smooth interpolation function $W\left(\boldsymbol{r}-\boldsymbol{r}^{\prime}, h\right)$ called the kernel as depicted in Fig. 1 (top). This function is called the kernel and it is defined on a compact support that depends on the smoothing length $h$. As shown by Monaghan (2005), this discretization scheme is second order accurate in space when the particles are arranged regularly in space. Then, Eq. 1 is applied to discrete particles by an integration quadrature. Hence, the function $f$ is expressed at the particle location $\boldsymbol{r}_{a}$ by:

$$
f\left(\boldsymbol{r}_{a}\right)=\sum_{b \in \Omega_{a}} V_{b} f\left(\boldsymbol{r}_{b}\right) W\left(\boldsymbol{r}_{b}-\boldsymbol{r}_{a}, h\right)
$$

where $V_{b}$ is the volume of particle $b$. The index $b$ refers to the neighbor particles located inside $\Omega_{a}$, the compact support centered around the particle $a$, as illustrated in Fig. 1 (bottom). In this work, the kernel is a quintic spline, given by:

$$
W(r, h)=\frac{1}{120 \pi h^{3}} \begin{cases}(3-r)^{5}-6(2-r)^{5}+15(1-r)^{5} & \text { for } 0 \leq r \leq 1 \\ (3-r)^{5}-6(2-r)^{5} & \text { for } 1<r \leq 2 \\ (3-r)^{5} & \text { for } 2<r \leq 3\end{cases}
$$

where $r=\left|\boldsymbol{r}_{a}-\boldsymbol{r}_{b}\right| / h$ is the normalized distance between particle $a$ and $b$. In the present study, the smoothing length $h$ is equal to the initial mean particle spacing $\Delta x$, and the radius of $\Omega$ is $R=3 h$. In the following, $f\left(\boldsymbol{r}_{a}\right), f\left(\boldsymbol{r}_{b}\right)$ and $W\left(\boldsymbol{r}_{b}-\boldsymbol{r}_{a}, h\right)$ are abbreviated by $f_{a}, f_{b}$ and $W_{a b}$, respectively.

The gradient and Laplacian operators are computed with the method proposed by Bonet and Lok (1999) and Cleary and Monaghan (1999): 


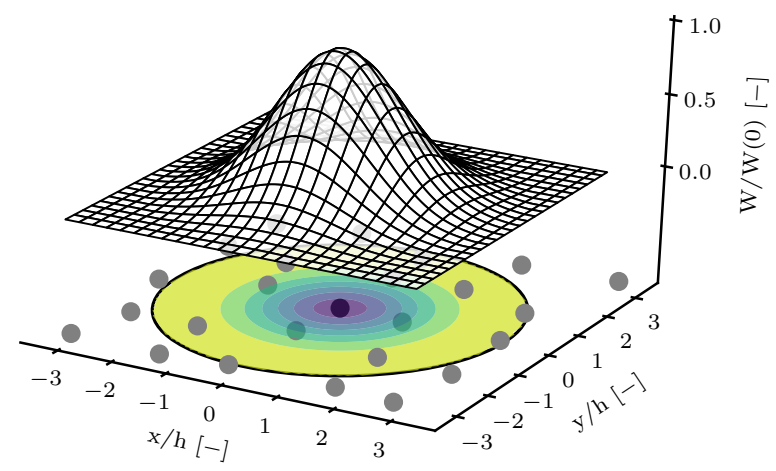

Figure 1: Top part: Surface of a 2-D kernel. Bottom part: Particle distribution superimposed with the kernel color map and illustration of the sphere of influence.

$$
\begin{aligned}
\nabla f_{a} & =\sum_{b \in \Omega} V_{b}\left(f_{b}+f_{a}\right) \nabla W_{a b} \\
\Delta f_{a} & =K \sum_{b \in \Omega} V_{b} \frac{f_{a}-f_{b}}{r_{a}-r_{b}} \nabla W_{a b}
\end{aligned}
$$

with $K=2(2+N), N$ being the number of geometrical dimensions in the simulation (Maciá et al., 2011).

\subsection{Governing Equations}

In this study, the Navier-Stokes equations of an isothermal flow are considered. Their local Lagrangian expression for a continuum is:

$$
\begin{aligned}
\frac{\mathrm{D} \rho}{\mathrm{D} t} & =-\rho \nabla \cdot \boldsymbol{u} \\
\rho \frac{\mathrm{D} \boldsymbol{u}}{\mathrm{D} t} & =-\nabla p+\mu\left(\Delta \boldsymbol{u}+\frac{1}{3} \nabla(\nabla \cdot \boldsymbol{u})\right)+\boldsymbol{g}-\sigma \kappa \boldsymbol{n} \delta_{\Sigma} \\
p & =\mathrm{F}(\rho)
\end{aligned}
$$

where $\mu$ is the kinematic viscosity of the fluid and $\boldsymbol{g}$ the gravity acceleration. The last term of Eq. 5b represents the force due to the surface tension, where $\sigma, \kappa$ and $\boldsymbol{n}$ represent the surface tension coefficient, the curvature of the phase interface and its normal vector, respectively. The term $\delta_{\Sigma}$ is called the surface delta function and was introduced by Brackbill et al. (1992) to convert a surface force to a volume force. The operator $(\nabla \cdot)$ is the divergence of a vector field, and the function $F$ in Eq. 5 c represents the Equation of State (EoS) that will be described in details in the following. Equations 5 are expressed in the SPH framework, for both the gas and liquid phase. The continuity equation is solved algebraically by computing the particle volume and density, as proposed by Español and Revenga (2003):

$$
\rho_{a}=m_{a} / V_{a} \quad \text { with } \quad V_{a}=1 / \sum_{b \in \Omega} W_{a b}
$$

where $m_{a}$ is the constant mass of particle $a$. Equations 6 exactly conserve mass. This expression of the density shows also the advantage of avoiding any numerical diffusion of density at the liquid/gas interface (Hu and Adams, 2006). The linear momentum balance on the particle $a$ is expressed by:

$$
\left.\rho_{a} \frac{\mathrm{d} \boldsymbol{u}}{\mathrm{d} t}\right|_{a}=\boldsymbol{f}_{a, p}+\boldsymbol{f}_{a, v}+\boldsymbol{f}_{a, g}+\boldsymbol{f}_{a, s t}
$$


(C)2019. This manuscript version is made available under the CC-BY-NC-ND 4.0 license http://creativecommons.org/licenses/by-nc-nd/4.0/

where $\boldsymbol{u}$ is the particle velocity and the terms $\boldsymbol{f}_{p, a}, \boldsymbol{f}_{v, a}, \boldsymbol{f}_{a, g}$ and $\boldsymbol{f}_{s t, a}$ are the forces due to pressure, viscosity, gravity and surface tension, respectively. They are given by:

$$
\begin{aligned}
\boldsymbol{f}_{a, p} & =-\sum_{b \in \Omega} V_{b}\left(p_{b}+p_{a}\right) \nabla W_{a b} \\
\boldsymbol{f}_{a, v} & =K \sum_{b \in \Omega} V_{b} \bar{\mu}_{a b} \frac{\boldsymbol{u}_{a b} \cdot \boldsymbol{r}_{a b}}{r_{a b}^{2}+\eta^{2}} \nabla W_{a b} \\
\boldsymbol{f}_{a, g} & =\boldsymbol{g} \\
\boldsymbol{f}_{a, s t} & =-\left(\sigma \kappa \delta_{\Sigma} \boldsymbol{n}\right)_{a}
\end{aligned}
$$

The viscosity model (Eq. 8b) was proposed by Cleary (1998), and conserves locally both linear and angular momentum. The inter-particle viscosity $\bar{\mu}_{a b}$ is derived from a combination of a harmonic mean of the densities and an arithmetic mean of the kinematic viscosities (Szewc et al., 2012):

$$
\bar{\mu}_{a b}=2 \frac{\rho_{a} \rho_{b}}{\rho_{a}+\rho_{b}} \frac{\nu_{a}+\nu_{b}}{2}
$$

where $\nu=\mu / \rho$ is the kinematic viscosity. This formulation was found to be more stable in our case. Equation $8 \mathrm{~b}$ features the scalar product of velocity difference $\boldsymbol{u}_{a b}=\boldsymbol{u}_{a}-\boldsymbol{u}_{b}$ by the inter-particle distance vector $\boldsymbol{r}_{a b}=\boldsymbol{r}_{a}-\boldsymbol{r}_{b}$. The term $\eta=0.1 \mathrm{~h}$ is added to avoid the singularity when the inter-particle distance goes to zero. The surface tension force (Eq. 8d) is an adaptation of the Continuum Surface Force (CSF) model (Brackbill et al., 1992) to SPH as proposed by Hu and Adams (2006) and improved by Adami et al. (2010).

The method presented here is based on the assumption that the fluids are weakly compressible. Hence, the pressure $p_{a}$ at particle $a$ is expressed by the equation of state originally proposed by Cole (1965):

$$
p_{a}=\frac{\rho_{0} c^{2}}{\gamma}\left[\left(\frac{\rho_{a}}{\rho_{0}}\right)^{\gamma}-1\right]+p_{b a c k}
$$

In Eq. 10, the terms $\rho_{0}, \gamma$ and $p_{b a c k}$ are the nominal particle density, the polytropic ratio and the background pressure, respectively. The term $c$ is the artificial speed of sound and must be chosen to satisfy $c \geqslant 10 u_{\max }$ in order to fulfill the weakly compressible condition by ensuring that density variation is lower than $1 \%$ (Monaghan, 1994). However, in the present case the Mach number of the gas phase is 0.17 . Thus, the weakly compressible assumption is not perfectly fulfilled, and the compressibility effects should be accounted for. As stated by Colagrossi and Landrini (2003), the use of Eq. 10 is able to describe an isothermal compressible flow. Considering the gas as an ideal gas, the ratio of total temperature over static temperature is given by:

$$
\frac{T_{t}}{T}=\frac{\gamma-1}{\gamma} \mathrm{Ma}^{2}+1
$$

and leads to a maximum increase of temperature of $0.8 \%$ which in turn leads to maximum decrease of density of $0.8 \%$, and an increase of viscosity of $0.5 \%$. Therefore the influence of the temperature variations on the density and the viscosity is negligible, and the flow can be considered as isothermal. In this case, the pressure is proportional to the density. Hence, in the simulation, the effective sound speed in the gas should be equal to the real one, so that Eq. 10 correctly describes the state of the gas. By defining the effective sound speed as $c_{e f f}=\sqrt{\partial p / \partial \rho}$ with the EoS defined in Eq. 10, one can show that:

$$
c_{\text {eff }}=\sqrt{\left(\frac{\partial p}{\partial \rho}\right)_{s}}=c\left(\frac{\rho}{\rho_{0}}\right)^{\frac{\gamma-1}{2}}
$$

Therefore setting $\gamma=1$ leads to $c=c_{\text {eff }}$, meaning that the artificial speed of sound is equal to the effective speed of sound. Hence, the artificial speed of sound in the gas $c_{g}$ is set equal to $340 \mathrm{~m} / \mathrm{s}$, and $\gamma_{g}$ to 1 . Concerning the speed of sound in the liquid, the classical assumption of weakly compressible flow is made 
to reduce the time-step. Assuming the maximum droplet velocity is the one of the gas, the maximum Mach number in the liquid is 0.03 , which ensures a weakly compressible flow. Therefore, the sound speed in the liquid can be reduced to obtain a larger time-step. Since the sound speed of the gas was already set to $340 \mathrm{~m} / \mathrm{s}$, it would not bring any benefit to set $c_{l}$ lower than $c_{g}$. Setting the sound speed of the liquid to $340 \mathrm{~m} / \mathrm{s}$ leads to a maximum Mach number of 0.17 , which is still satisfactory for the present application. The polytropic ratio $\gamma_{l}$ is classically set to 7 to mimic the incompressibility of water. Note that, as shown later, the time-average of the droplet velocity reaches a maximal value of $40 \mathrm{~m} / \mathrm{s}$, leading to a Mach number of 0.11 , which perfectly fulfills the assumption of weakly-compressible flow.

Since $c_{g}=c_{l}$, the ratio $\rho_{l, 0} c_{l}^{2} / \rho_{g, 0} c_{g}^{2}$ is equal to $\rho_{l, 0} / \rho_{g, 0} \approx 93$. In our code, it was observed that a large $\rho_{l, 0} c_{l}^{2} / \rho_{g, 0} c_{g}^{2}$ ratio leads to numerical instabilities at the interface. This is explained by the calculation of the pressure gradient in the vicinity of the interface. Considering one elementary contribution between a gaseous particle $a$ and a liquid particle $b$ in the pressure gradient:

$$
\mathrm{d} \boldsymbol{f}_{a, p}=-V_{b}\left(p_{b}+p_{a}\right) \nabla W_{a b}
$$

We consider a small density variation for particle $a$ and particle $b$, so that $\rho / \rho_{0} \sim 1+\epsilon$, with $\epsilon \ll 1$. In addition we suppose this variation of the same order of magnitude, so that $\epsilon_{a} \sim \epsilon_{b} \sim \epsilon$. Expressing the pressures by the EoS (Eq. 10), Eq. 13 reads:

$$
\mathrm{d} \boldsymbol{f}_{a, p}=-V_{b}\left[\left(\rho_{g, 0} c_{g}^{2}+\rho_{l, 0} c_{l}^{2}\right) \epsilon+2 p_{b a c k}\right] \nabla W_{a b}
$$

We suppose that the effect of background pressure is negligible versus the effect of compressibility, so that we can drop $p_{\text {back }}$ in Eq. 14. In our case, since $\rho_{l} c_{l}^{2} \gg \rho_{g} c_{g}^{2}$, we can write:

$$
\mathrm{d} \boldsymbol{f}_{a, p} \sim-V_{b} \rho_{l, 0} c_{l}^{2} \epsilon \nabla W_{a b}
$$

which means that the contribution of the pressure gradient at particle $a$ due to the interaction between $a$ and $b$ is mainly imposed by the density at particle $b$. In other words, close to the interface and in case of a large density ratio, the pressure gradient in the light phase is determined by the properties and the density field of the heavy phase. Also, due to the prefactor $\rho_{l, 0} c_{l}^{2}$, the amplitude of the pressure gradient in the light phase is too large with regard to the particle density. This triggers a noisy interface, which leads to numerical instabilities. To circumvent this effect, one can impose $\rho_{l, 0} c_{l}^{2} \approx \rho_{g, 0} c_{g}^{2}$ as done by Chaussonnet et al. (2018). However in our case, this can lead to a liquid Mach number close to one, which is not satisfactory. Therefore, the term $\left(p_{a}+p_{b}\right)$ in Eq. 8a is replaced by a blending function:

$$
2 \bar{p}+\Delta p \frac{d_{b}-d_{a}}{3 h} \quad \text { with } \quad \bar{p}=\frac{p_{a}+p_{b}}{2} \quad \text { and } \quad \Delta p=\frac{p_{b}-p_{a}}{2}
$$

The terms $d_{a}$ and $d_{b}$ are the distances from the particle to the interface, as depicted in Fig. 2. This correction is applied only in the transition band i.e. within a distance of $3 h$ to the interface. This tends to decrease the influence of the heavy phase for particles of the light phase and vice versa.

The resulting set of equations (Eqs. 6-10) is integrated in time by means of a predictor-corrector scheme. The time step of the numerical simulation is controlled by convection, viscosity, surface tension and the overall acceleration. It is computed at each iteration step:

$$
\Delta t=\min _{a}\left(C_{1} \frac{h}{\left\|\boldsymbol{u}_{\boldsymbol{a}}\right\|+c}, \quad C_{2} \frac{h^{2}}{\nu_{a}}, \quad C_{3} \sqrt{\frac{\rho_{a} h^{3}}{2 \pi \sigma_{a}}}, \quad C_{4} \sqrt{\frac{h}{\Gamma_{a}}}\right)
$$

where $\Gamma_{a}$ is the magnitude of the particle acceleration and $C_{1}$ to $C_{4}$ are constants equal to $0.5,0.25,0.25$ and 0.5 , respectively. 
(C)2019. This manuscript version is made available under the CC-BY-NC-ND 4.0 license http://creativecommons.org/licenses/by-nc-nd/4.0/

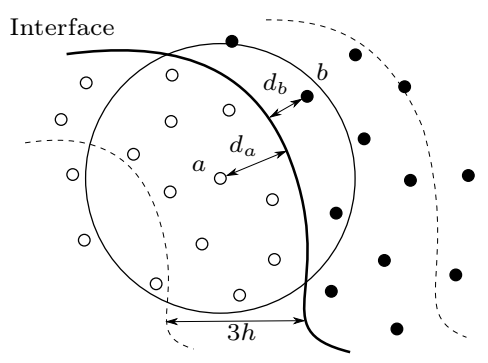

Figure 2: Sketch of the interface and the transition band in vicinity of the interface.

\subsection{Boundary conditions}

In the SPH method, inlet and outlet boundary conditions are of permeable types. New particles are injected at the inlet and deleted at the outlet of domain. In the method presented here (Fig. 3), a layer of particle is defined upstream the inlet and downstream the outlet to provide a complete compact support of the kernel to the particles located at boundaries. This layer is called the buffer zone. To these particles the prescribed physical boundary conditions, namely the velocity at the inlet and the pressure at the outlet are imposed. The computational domain is virtually delimited by the markers (Fig. 3) at the boundaries. When the particles cross the boundaries, their status switch from buffer to regular and they are treated according to Eqs. 6-10. Pressure waves are damped by applying the method presented in (Braun et al., 2015a). Walls are modeled with several layers of particles to complete the sphere of influence of the kernel, and a no-slip condition is imposed (Takeda et al., 1994). A detailed discussion of boundary conditions for the SPH method is available in (Braun et al., 2015a).

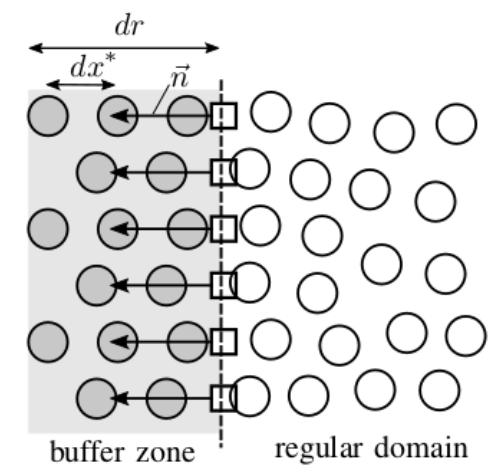

Figure 3: Buffer (gray) and regular (white) particles separated by markers (squares), from Braun et al. (2015a).

\subsection{Post-processing tools for the liquid phase}

The post-processing tools applied to the liquid phase are the important final step that ensures the transfer of knowledge from the raw numerical simulation to formatted results exploitable by a broader audience. These results can then be directly compared to the experiment or can be used to derive parameters that characterize the investigated phenomenon. For instance, in the present case, a critical output of the predicted atomization is the Sauter Mean Diameter (SMD), which is equivalent to the mean diameter of the spray droplets if the overall volume-to-surface ratio was kept constant. This quantity characterizes the efficiency of the spray generation and hence the performance of the atomizer. It can be used also as boundary conditions for a large-scale simulation that focuses on the phenomena downstream the atomization (e.g. evaporation, turbulent mixing and/or combustion). In this subsection we introduce the different steps of the post-processing chain, whose overview is presented in Fig. 4. 
(c)2019. This manuscript version is made available under the CC-BY-NC-ND 4.0 license http://creativecommons.org/licenses/by-nc-nd/4.0/

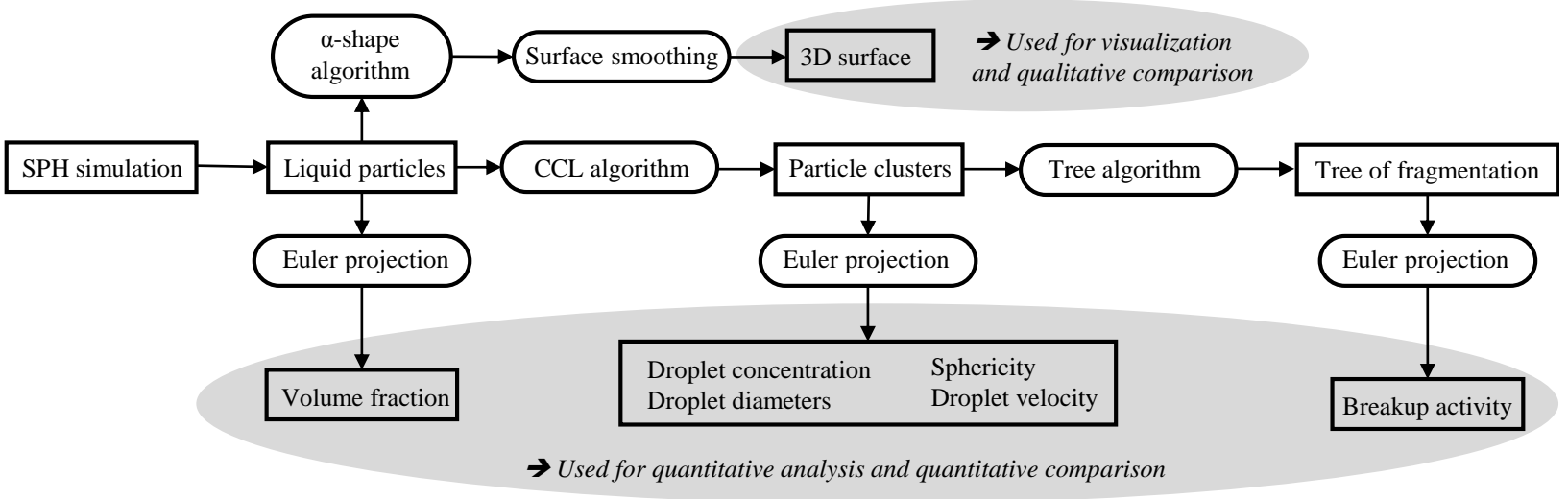

Figure 4: Overview of the tool chain for the post-processing of the liquid phase.

In a first step, the liquid particles are separated from the wall and the gas particles, which reduces the size of the dataset by $99.71 \%$ in the present case. From this dataset a 3D surface of the liquid phase can be extracted with the $\alpha$-shape algorithm (Edelsbrunner et al., 1983). With this method, the liquid surface is directly tessellated from the utmost liquid particles. In order to reduce the aliasing effect and provide more realistic liquid structures, an additional step of smoothing is applied to the surface, which can lead to a significant reduction of the liquid volume. Then, the 3D surface can be used for qualitative analysis, or qualitative comparison with experiments, when the volume reduction is not critical. A more detailed comparison of different techniques for surface representation in SPH was published previously by Braun et al. (2016b).

In order to quantitatively analyze the data, additional post-processing tools are applied. The liquid particles can be directly projected on a background mesh (Eulerian projection). This projection allows (i) comparisons with mesh-based methods and (ii) to compute the time-averaged quantities, such as the volume fraction, the momentum or the energy of the liquid phase.

To access the spray characteristics, a Connected Component Labeling (CCL) technique (Rosenfeld and Pfaltz, 1966) is applied. This technique allows to assign to each cluster of numerical particles as a physical equivalent droplet. The position, the velocity and the volume of each cluster is calculated from the corresponding values of its numerical particles, and the diameter of equivalent volume can be determined. In addition, the sphericity $\varphi$ of the cluster, defined as illustrated in Fig. 5, can be determined. These quantities

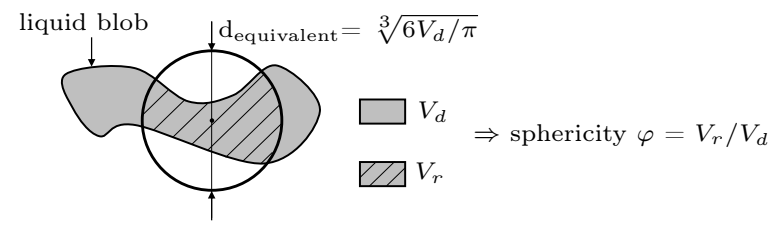

Figure 5: Definition of sphericity $\varphi$. The sketch is in 2D for the sake of simplicity. The circle of equivalent volume is centered at the center of gravity of the liquid blob.

can be used directly in their Lagrangian form or they can be projected on a mesh to be time-averaged, and to give access to the liquid volume concentration. It is to be emphasized that in the present study, clusters that contain less than 5 particles are dismissed due to the limited spatial resolution. This is equivalent to dismiss droplets smaller than $2 \mathrm{dx}$.

Finally, by exploiting the Lagrangian aspect of the SPH method, it is possible to build the tree of fragmentation from the set of the particle clusters. The tree of fragmentation is defined here as a tree object that contains all the relationships between the mother and children droplets, as illustrated in Fig. 6. In addition 
to the mother/children relationships, the information of position, time, velocity, diameter and sphericity is attributed to each droplet. The droplets are identified by a hash table built from the md5sum algorithm (Rivest, 1991) applied to the concatenation of the particle IDs that compose the droplet. Therefore, it is necessary to attribute a unique ID number to particles when they are injected into the domain. This procedure is straightforward due to the Lagrangian character of the SPH method. During their motion across the numerical domain, a particle cluster that does not breakup is always composed of the same numerical particles, which lead to the same hash key.

From this tree of fragmentation it is possible to extract various quantities like the number of breakup
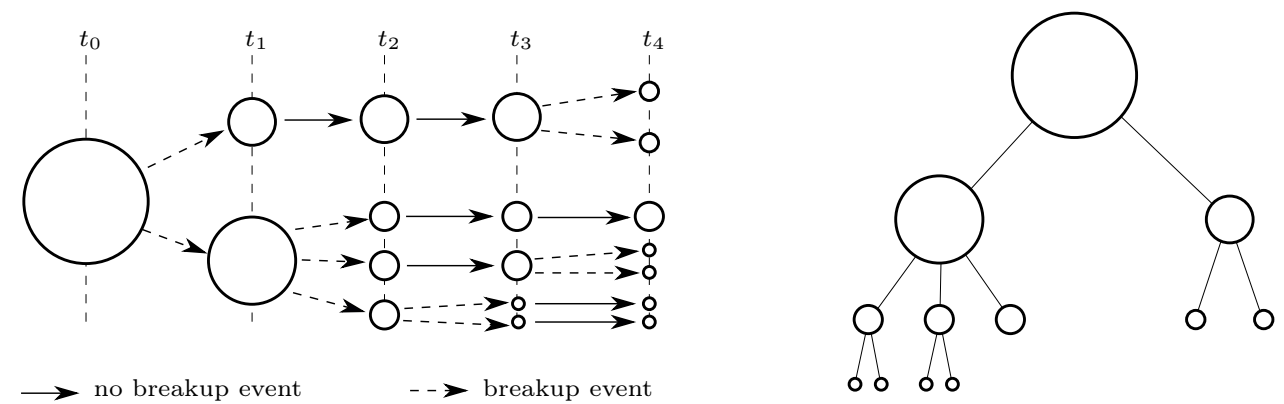

Figure 6: Time evolution of the breakup of a single droplet (left) and its corresponding tree of fragmentation (right).

events that a droplet undergoes before exiting the domain, or the distribution of the $d_{c h i l d} / d_{m o t h e r}$ ratio, also referred to as the fragmentation intensity spectrum (Gorokhovski and Saveliev, 2008). The position $\mathbf{r}_{b u}$ of the breakup event is defined as the mass-weighted barycentric mean position of the mother drop and the children:

$$
\mathbf{r}_{b u}=\frac{(m \mathbf{r})_{\text {mother }}+\sum_{\text {children }} m \mathbf{r}}{m_{\text {mother }}+\sum_{\text {children }} m}
$$

The time of breakup event $t_{b u}$ is the average of the last time of the mother and the first time of the children. For instance, considering the breakup events between $t_{0}$ and $t_{1}$ in Fig. 6 , the time $t_{b u}$ of the breakup event is $t_{b u}=\left(t_{0}+t_{1}\right) / 2$. By projection of all breakup events on a background grid, it is possible to define the breakup activity $N_{\phi}$ that corresponds to the number of breakup events per volume and per time unit.

\section{Reference experiment}

In this section we describe the reference experiment that will be used to validate the simulation. It was conducted by Sänger et al. (2015) on the Pressurized Atomization Test-rig (PAT) (Jakobs et al., 2015). The experiment consists of a twin-fluid external mixing nozzle as depicted in Fig. 7. The liquid emerges with low velocity in the form of a cylindrical jet, and the gas forms an annular coaxial high speed stream enclosing the liquid. The geometric parameters are the liquid diameter $D_{l}=2 \mathrm{~mm}$, the gas height $H_{g}=1.6 \mathrm{~mm}$, the separator thickness $e_{s}=0.1 \mathrm{~mm}$ and the gas diameter $D_{g}=5.4 \mathrm{~mm}$.

The atomization process is achieved by the momentum transfer from high-speed gas phase to the slow liquid phase. The liquid is a mix of Glycerol and water. Its characteristics are a density $\rho_{l}$ of $1233 \mathrm{~kg} / \mathrm{m}^{3}$, a constant dynamic viscosity $\mu_{l}$ of $0.2 \mathrm{~Pa} \mathrm{~s}$, and a surface tension $\sigma$ of $63.6 \mathrm{mN} / \mathrm{m}$. The reactor is pressurized up to 11 bar and the temperature is kept at $20^{\circ} \mathrm{C}$, leading to density $\rho_{g}$ of $13.25 \mathrm{~kg} / \mathrm{m}^{3}$ and a dynamic viscosity $\mu_{g}$ of $18.61 \mu \mathrm{Pa}$ s. The mean gas velocity is $58 \mathrm{~m} / \mathrm{s}$. The non-dimensional numbers that characterize this experiment are:

$$
\operatorname{Re}=\frac{\rho D_{h} U}{\mu}, \text { We }=\frac{\rho_{g} D_{l} U_{r e l}^{2}}{\sigma}, \mathrm{Oh}=\frac{\mu_{l}}{\sqrt{\sigma \rho_{l} D_{l}}}
$$




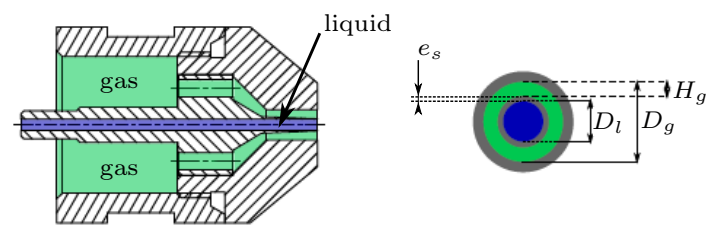

Figure 7: Schematics of external mixing twin fluid atomizer, side view (left) and front view (right).

Equations 19 refers to, in order of appearance: the Reynolds number where $D_{h}$ is the hydraulic diameter equal to $D_{l}$ for the liquid and $2 H_{g}$ for the gas, the Weber aerodynamic number where $U_{r e l}$ is the liquid/gas relative velocity, and the Ohnesorge number. In the present study, the Reynolds numbers are 10.5 and 137000 for the liquid and the gas, respectively. The Weber number is 1375 and the Ohnesorge number is 0.048. Additionally, two operating parameters are introduced. First, the gas-to-liquid mass ratio (GLR) determines the mass flux ratio of the two phases injected in the domain and second, the momentum flux ratio $M$ is a measure of the momentum transfer from gas to liquid. They are expressed as:

$$
\mathrm{GLR}=\frac{\dot{m}_{g}}{\dot{m}_{l}} \quad \text { and } \quad \mathrm{M}=\frac{\rho_{g} U_{g}^{2}}{\rho_{l} U_{l}^{2}}
$$

and are equal to 4.4 and 49 , respectively. They corresponds to a gas and liquid mass flow rate of 14.8 and $3.3 \mathrm{~g} / \mathrm{s}$.

The liquid disintegration was recorded in the experiment by a high-speed camera at a sampling frequency of $12 \mathrm{kHz}$. It was observed that the jet can feature different types of global behavior (pulsating or flapping mode) depending on liquid viscosity and GLR. These modes are illustrated in Fig. 8(a) and (b). As expected, the ambient pressure has been found to affect the type of breakup regime. At lower ambient pressure the liquid disrupts in a membrane mode (Fig. 8b and c) where the liquid forms thin membranes in a similar way like a bag breakup. When the pressure increases, the liquid is fragmented by a fiber-type breakup where the liquid is fragmented into smaller stretched lumps (Fig. 8d). The regime corresponding to operating conditions of the present simulation is the fiber-type breakup.
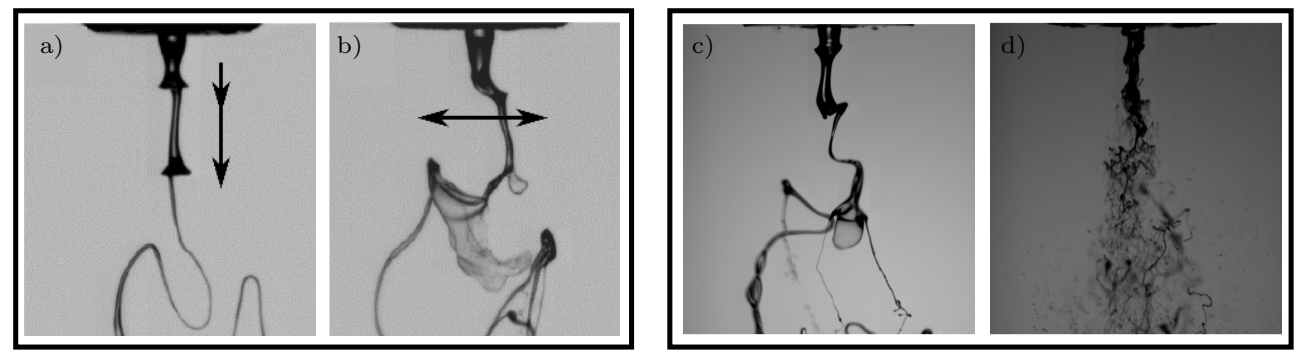

Figure 8: Primary instabilities of a liquid jet. Pulsating mode (a) at $\mu_{1}=200 \mathrm{mPa}$ s and flapping mode (b) at $\mu_{1}=$ $300 \mathrm{mPa}$ s, from Sänger et al. (2014). Membrane-breakup (c) at 1 bar (GLR = 0.36) and fiber-breakup (d) at 7 bar $(\mathrm{GLR}=2.5)$, from Sänger et al. $(2015)$

The primary instability that is responsible for the liquid fragmentation is of Kelvin-Helmholtz type. The frequency of this instability was measured by a Fourier Transform of the time signal obtained from the pixel values of a zone located in the vicinity of the jet. It was measured to be $2153 \mathrm{~Hz}$.

The intact length is defined here as the distance where the liquid jet disrupts and all the structures downstream are completely detached from it. The intact length was estimated by contour recognition based on a threshold pixel value. This threshold $K_{p}$ was determined by the maximum entropy method by Kapur et al. (1985). However, due to the three-dimensional distortion of the liquid jet surface located out of the 
(C)2019. This manuscript version is made available under the CC-BY-NC-ND 4.0 license http://creativecommons.org/licenses/by-nc-nd/4.0/

Depth-of-Field, the determination of the intact length is not straightforward, and it highly depends on the value of the threshold determined by the maximum entropy method, as illustrated in Fig. 9.

Therefore to investigate the dependence of the intact length on the threshold, the threshold $K_{p}$ was multiplied by a factor $F$ that varies from 0.01 to 2 (Fig. 10). It is observed that the mean value of the critical length is linearly dependent on $F$ up to 1 .

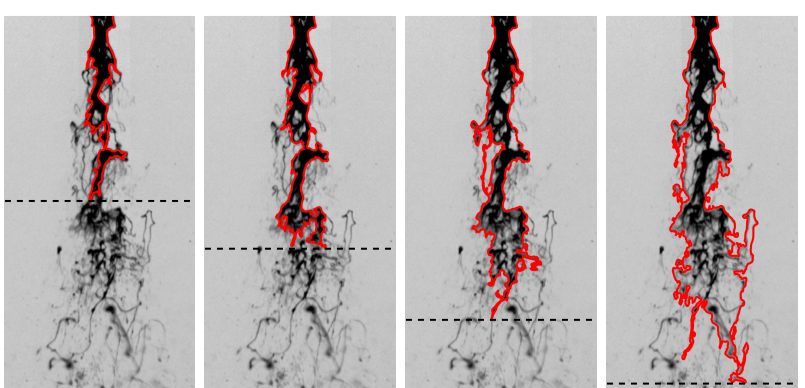

Figure 9: Influence of the threshold on the determination of the intact length. From the left to the right, the threshold is $10,50,75$ and $100 \%$ of $K_{p}$.

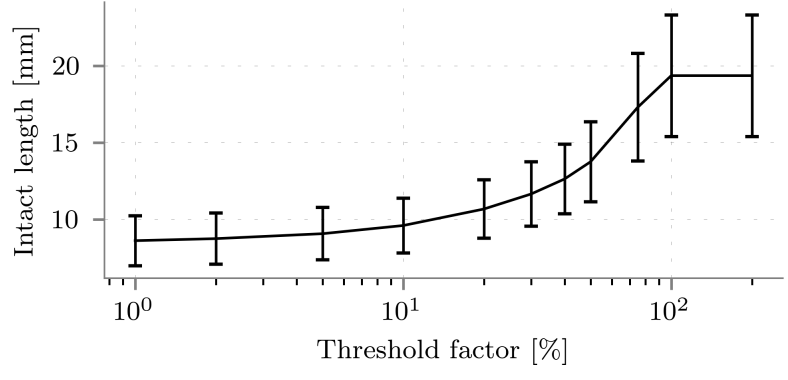

Figure 10: Intact length versus $F$.

\section{Numerical Setup}

The twin fluid atomizer, shown in Fig. 7 is modeled by means of SPH. In order to reduce the computational expenses, the numerical domain, depicted in Figs. 11 and 12, is simplified to the nozzle and a fraction of the pressurized cavity. The pressurized cavity is reduced to the vicinity of the nozzle, in the form of a cylinder whose length and diameter are 30 and $17.4 \mathrm{~mm}$, respectively (Fig. 12). Because of this geometrical reduction, a small amount of liquid lumps will exit the domain without being completely fragmented, as it will observed in Sections 6.2 and 6.5. However, this does not modify the fundamental breakup process that occurs in the nozzle vicinity.

The channel lengths of the coaxial gas and liquid streams are equal to $6 H_{g}$ and $D_{l}$, respectively. A turbulent profile is set at the inlet of the gas channel, whereas a laminar profile is set at the liquid channel. The wall height $H_{n}$ of the outer crown of the nozzle is limited to $3 \mathrm{~mm}$. On the walls of the nozzle a no-slip condition is imposed. In order to take the entrainment rate of the coaxial jet into account, an additional gas inlet of 3 $\mathrm{mm}$ height is added on the outer radius of the cavity. The entrainment velocity is then $14.84 \mathrm{~m} / \mathrm{s}$ and ensures an appropriate entrainment rate to the outlet of the cavity (Hussein et al., 1994). On the side of the cavity a wall without friction is set as boundary condition. Finally, the outlet is set as a free flow condition with a constant pressure of 11 bar. Note that the elevated ambient pressure is taken into account in the gas density only. Figures 11 and 12 also show the virtual probes that will be referred to in the following. The rings in the near-nozzle region (Fig. 11) will be used to detect the Kelvin-Helmholtz instability on the surface of the jet while the spray characteristics will be determined on the two slices depicted in Fig. 12. The mechanical properties of the gas and liquid are the same as in the experiment. The background pressure ( $p_{b a c k}$ in Eq. 10) was limited to $27.5 \mathrm{kPa}$. This value was determined as the minimum value to avoid the formation voids in the particle lattice, especially at the center of large-scale vortices. When the background pressure is normalized by the $\rho c^{2}$, it is equal to 0.18 and 0.002 for the gas and liquid phase, respectively. Since theses values are significantly lower than one, it is ensured that the detrimental effect of the background pressure on the particle motion will have a limited effect.

The initial inter-particle spacing is equal to $33.33 \mu \mathrm{m}$, which leads to 208 million particles and 370 thousand markers. At the initiation of the simulation the cavity is filled with gas particles and the liquid jet is initialized as a $5 \mathrm{~mm}$ long cylinder. The simulation is run on 2000 cores for a physical time of 45 $\mathrm{ms}$, equivalent to 87 gaseous convective times. With an initial transient state of the liquid jet of $6 \mathrm{~ms}$, it leads to $39 \mathrm{~ms}$ (76 convective times) of physical time to collect statistics on the liquid phase. During this 


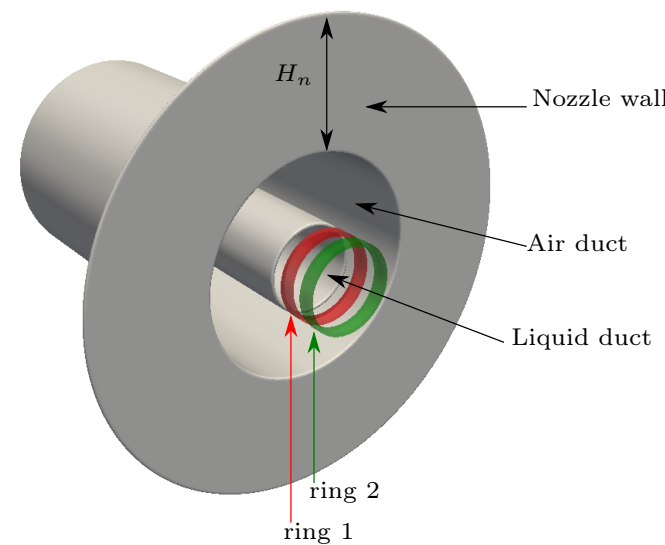

Figure 11: Close-up view of the nozzle, superimposed with the virtual rings.

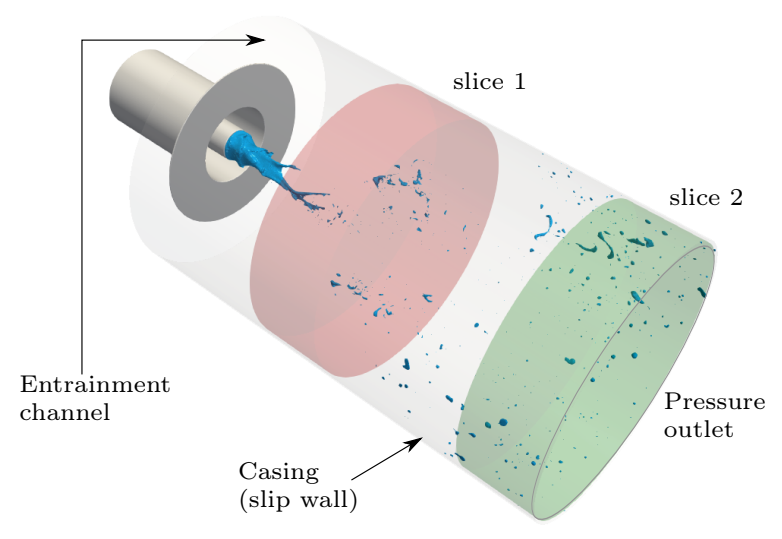

Figure 12: Numerical domain superimposed with the virtual slices.

period, a large number of breakup events are resolved, which ensures an appropriate convergence of the spray statistics. With a mean time step $\Delta t$ of $28 \mathrm{~ns}$, the simulation is run over 1.6 million of iterations. This amounts to a total computational cost of 1.2 millions CPU-hours. In total 1553 time step were exported, corresponding to a sampling frequency of $34.5 \mathrm{kHz}$.

\section{Qualitative results}

In this section, the surface of the liquid phase is extracted with the $\alpha$-shape method presented in Section 2.4. The animation ${ }^{1}$ of the simulation showed that most of the time, the liquid jet is peeled off and fragmented axially. However, some flapping events where the jet is radially deflected were also observed. This intermittent behavior is also observed in the experiment.

A flapping event is qualitatively compared to the high-speed camera measurements in Fig. 13. Time series of both the experiment and the simulation are placed side by side. The time series start at an arbitrary time $t$. The simulation captures well the flapping instability of the liquid jet observed in the experiment. Moreover, the length scales of the hook-shape of the liquid jet in the simulation reflects well the high-speed images. Also, the time series highlight that the temporal evolution of the liquid hook matches the experiment. Nevertheless, in the simulation, the spray shows a lower volume fraction of liquid than in the experiment, especially at the tip of the liquid jet. Three reasons were identified to explain this discrepancy. First, the starting conditions are not exactly the same, because the liquid contained at the tip of the jet at $t=0 \mu \mathrm{s}$ is already larger in the experiment. Second, droplets containing less than 5 particles were discarded because of a too low resolution that would not render a realistic behavior. In addition the presence of very small droplets would hide the ligaments, which would decrease the clarity of Fig. 13. Discarding the under-resolved droplets leads to a significant overall reduction of the spray volume. Third, the surface smoothing procedure further reduces the volume of the liquid volume.

Subsequently, a single liquid lump from the simulation is compared to a similar lump taken from the experiment in Fig. 14. Snapshots of the simulation and the experiment are disposed side by side. In order to avoid any volume reduction of the spray, the structure is presented as a raw simulation output, in the form of a particle cluster. The liquid lump shows the same shape evolution with a mushroom like structure (i). Then, the cap of the mushroom is sheared, leading to ligaments elongated in the streamwise direction (ii). The shape of the structure resembles the contour of a jellyfish. Finally, the ligaments are further elongated to thin threads and the cap of the mushroom is reduced in size (iii).

\footnotetext{
${ }^{1}$ Accessible on our Youtube channel SPH@ITS: https://www . youtube.com/watch?v=Ld4nRMHz7MQ\&t=12s
} 
(C)2019. This manuscript version is made available under the CC-BY-NC-ND 4.0 license http://creativecommons.org/licenses/by-nc-nd/4.0/

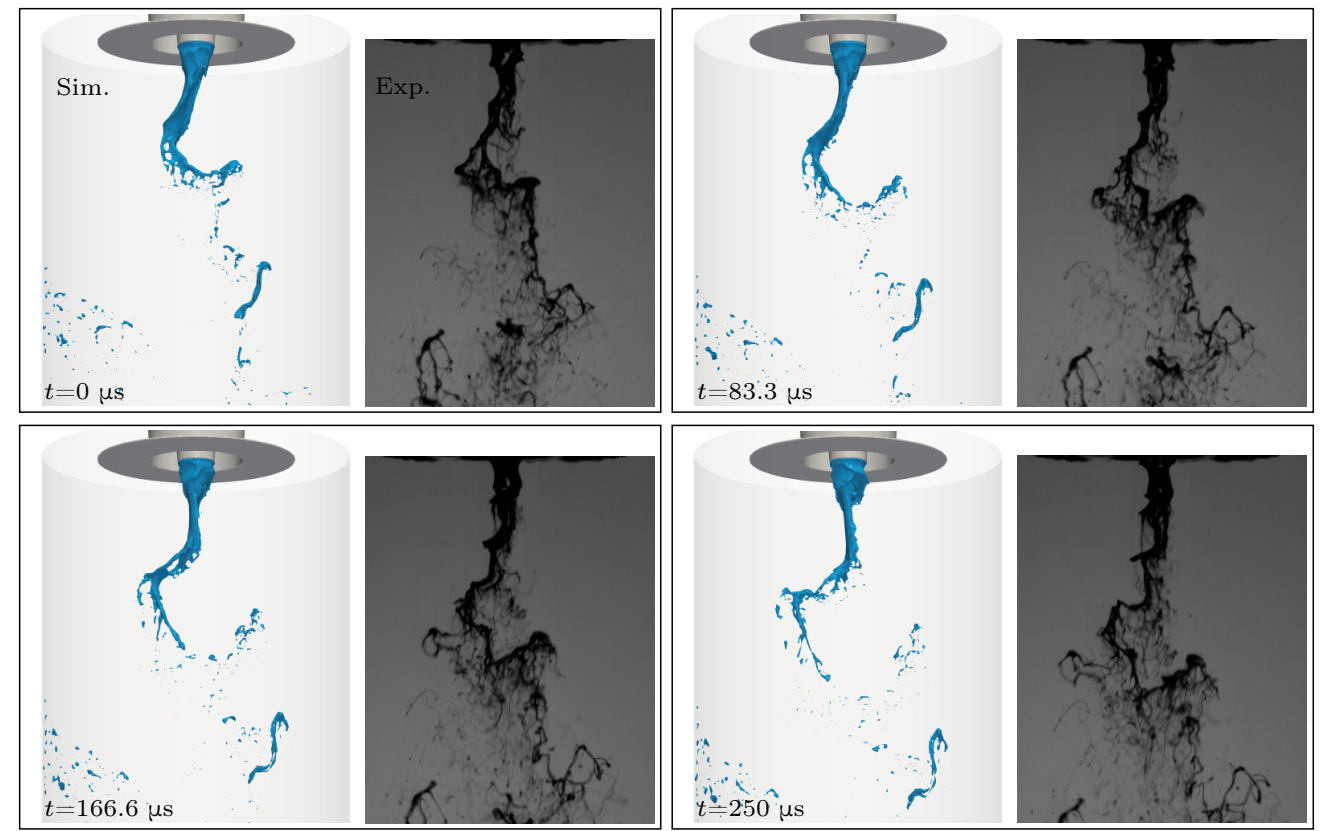

Figure 13: Comparative time series of a flapping event, starting at an arbitrary time $t$.

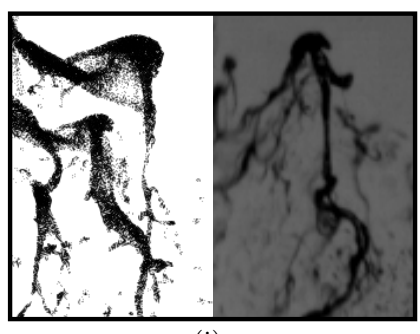

(i)

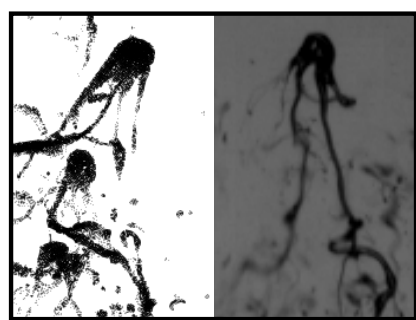

(ii)

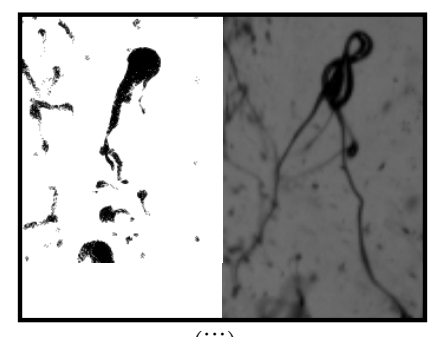

(iii)

Figure 14: Comparison of a liquid lump torn from the jet and being further fragmented. Simulation (left) and experiment (right) are disposed side by side.

Finally, the 3D effects of the flapping instability are illustrated in Fig. 15. The top row shows a flapping event on an arbitrary view plane, whereas the bottom row shows the same event with a view plane perpendicular to the first one. The flapping event is clearly observable on the top row while it is not on the bottom row. This figure illustrates the difficulty to record a flapping motion in 3D when the flapping axis constantly rotates, with only one camera. Depending on the orientation of the flapping motion, the camera can capture it only if it is adequately oriented (top row of Fig. 15). If the camera is not appropriately oriented, the flapping motion is not distinguishable (bottom row of Fig. 15). In the numerical simulation, this result is directly accessible whereas it requires the installation of additional synchronized high-speed cameras in the experiment. This larger flexibility illustrates one advantage of 3D simulation over experimental measurement for the $3 \mathrm{D}$ characterization of liquid structures. 

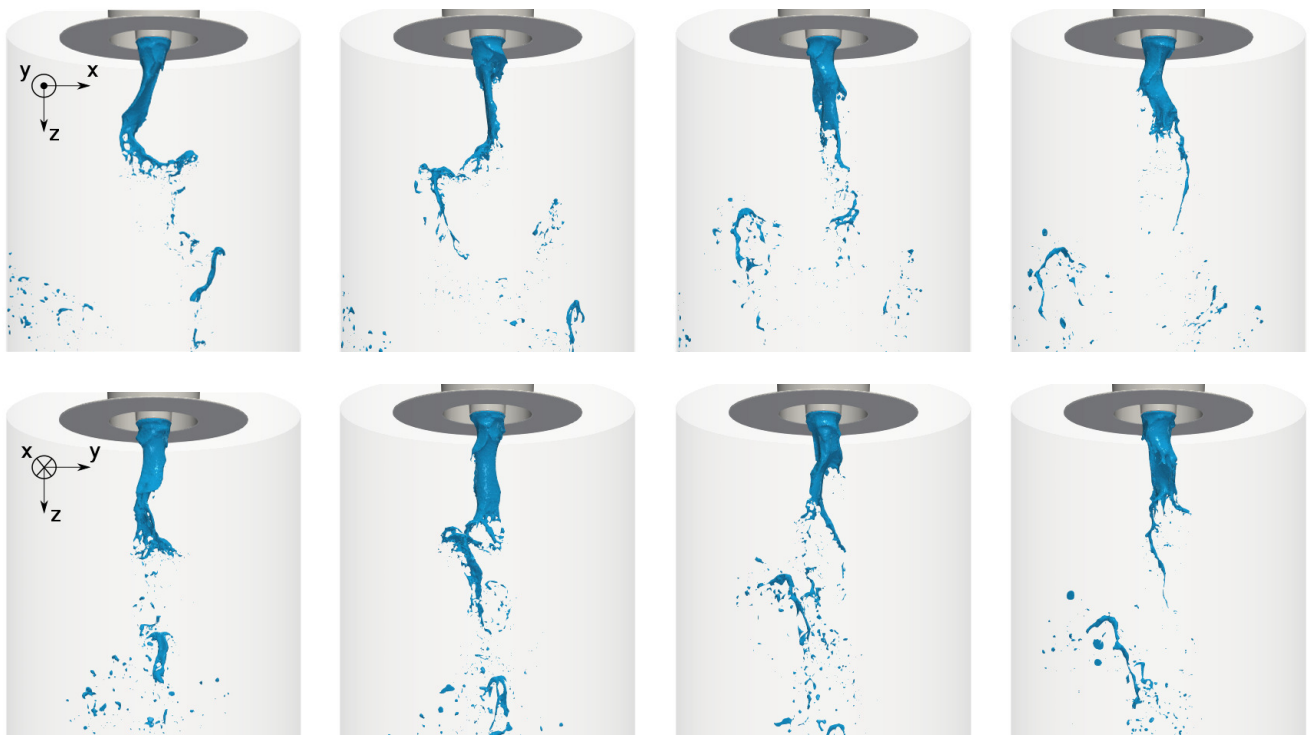

Figure 15: Time series of liquid breakup with $\Delta \mathrm{t}=0.29 \mathrm{~ms}$. Front view (top) and side view (bottom).

\section{Quantitative results}

\subsection{Detection of the Kelvin-Helmholtz instability and the dynamics of the intact length}

In the simulation, the Kelvin-Helmholtz instability was detected by the two annular probes located at the exit of the liquid channel, labeled ring 1 and ring 2 in Fig. 11 . The probes are located directly at the nozzle exit, similarly to the location of the pixels of the high-speed camera that were analyzed from the experiment. In the volume enclosed by each probe, the liquid volume fraction was monitored during the simulation, and plotted versus time. For instance, if the volume of one probe was full of liquid on a given time period $\Delta t$, the probe would output a volume fraction of 1 during $\Delta t$. Therefore, at the end of the simulation, these probes provide a time signal of the volume fraction. A Fourier Transform was applied to the signal provided by the two probes, and the Cross Spectral Density (CSD) was computed (Fig. 16). Blackman sampling windows as well as zero padding were used. This gave a frequency resolution of $5.7 \mathrm{~Hz}$. The peak in the FFT was found at $2319 \mathrm{~Hz}$ which is to be compared to $2153 \mathrm{~Hz}$ of the experiment, leading to a deviation of $6.7 \%$.

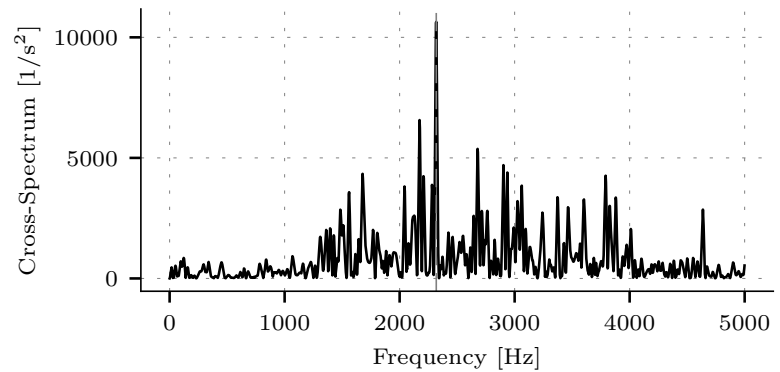

Figure 16: Cross Spectral Density of the liquid volume fraction time signal in probes ring 1 and ring 2 as depicted in Fig. 11.

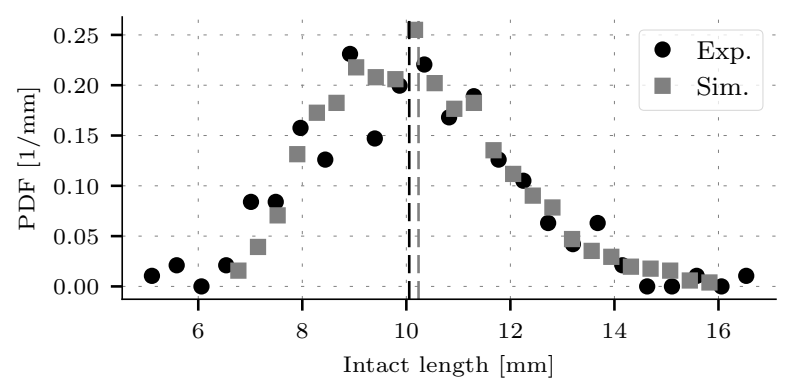

Figure 17: Probability density functions of the jet intact length. Dashed line indicates the mean value.

The liquid jet is detected using the CCL method (Rosenfeld and Pfaltz, 1966) as the largest particle cluster. The intact length is calculated as the longest axial extent of the liquid jet, and it was extracted 
(C)2019. This manuscript version is made available under the CC-BY-NC-ND 4.0 license http://creativecommons.org/licenses/by-nc-nd/4.0/

for each time step. Its Probability Density Function (PDF) is plotted in Fig. 17, superimposed with the PDF of the intact length extracted from the experiment, for a threshold $F$ of $20 \%$ of the median pixel value. This threshold was selected in order to obtain the same mean value, i.e. a PDF centered at the same location. The agreement of the shape of the PDF, as well as its width is good. This indicates that the present method is successful in capturing not only the mean of the intact length but also its fluctuations, in terms of distribution and extreme values. To conclude this subsection, we have shown here that the code is able to predict the dynamics of the macroscopic phenomena related to the liquid phase, in terms of KelvinHelmholtz frequency and fluctuations of the intact length. Note that it is observed that single droplets are regularly torn off from the central jet during the simulation. However, they represent a negligible amount of liquid, so that they do not influence the dynamics of the jet.

\subsection{Maps of time-averaged liquid quantities}

In order to extract the time average of liquid physical quantities, the numerical particles of the liquid type were projected on a half infinite plane corresponding to the radial and axial coordinates $(r, z)$. It is illustrated in Fig. 18 in immersion in the geometry (left) and in the form where the results will be presented (right). This projection allows to obtain the instantaneous $2 \mathrm{D}$ map of liquid fraction $\alpha_{L}(r, z, t)$ in the cavity according to:

$$
2 \pi r \alpha_{L}(r, z, t) \mathrm{d} r \mathrm{~d} z=\sum_{p \in d V(r, z)} V_{p} \Omega_{p}
$$

where $V_{p}$ is the particle volume. The term $d V(r, z)$ represents the elementary crown volume of radius $r$ located at $z$, as illustrated in Fig. 18. The section of this crown is $\mathrm{d} S=\mathrm{d} r \mathrm{~d} z$. The term $\Omega$ is a weight
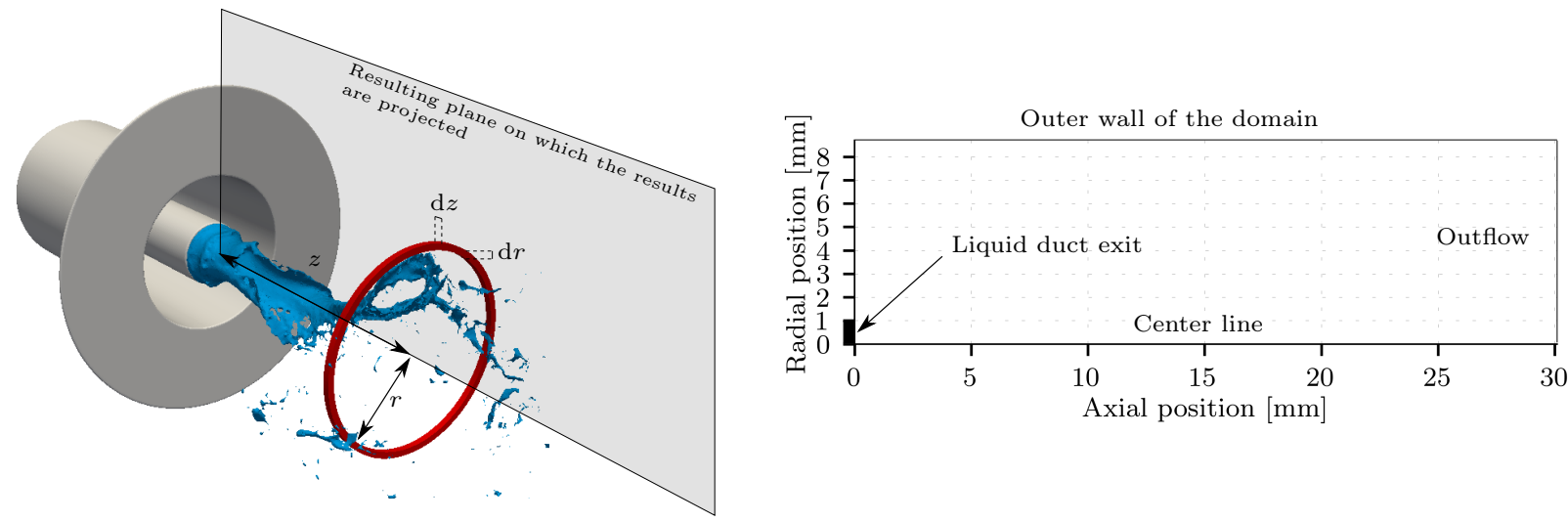

Figure 18: Left: Illustration of the elementary crown $d V(r, z)$ and the resulting plane of projection. Right: Projection plane with features of the numerical setup.

factor that accounts for the velocity of the liquid phase. Due to the large number of samples contained in a restricted averaging period, two consecutive time frames might be correlated in terms of liquid presence. Therefore slow particles would be counted more often than fast particles. In order to counterbalance this bias, the factor $\Omega$ is proportional to the velocity of the liquid phase, and is expressed as:

$$
\Omega_{p}=C \frac{\|\mathbf{U}\| \mathrm{d} t}{\sqrt{\mathrm{d} r \mathrm{~d} z}}
$$

where $C$ is a constant ensuring that $\sum_{p \in d V(r, z)} \Omega_{p}=1$. Then, the instantaneous volume fraction is timeaveraged to obtain the mean quantity plotted in Fig. 19a. The quantities extracted by the CCL method were used to draw the maps of droplet concentration, Sauter Mean Diameter and sphericity, according to an expression similar to Eq. 21. Their fields are shown in Fig 19b to 19d. In total, 2.372 million droplets were collected between $\mathrm{t}=6$ and $45 \mathrm{~ms}$. 
(C)2019. This manuscript version is made available under the CC-BY-NC-ND 4.0 license http://creativecommons.org/licenses/by-nc-nd/4.0/

In Fig. 19a, the plotted volume fraction map is typical for air-assisted atomization. The axial extent of the main core of the liquid jet $\left(\alpha_{L} \approx 1\right)$ is $2.5 \mathrm{~mm}$, to be compared to the law by Lasheras et al. (1998) $6 D_{l} / \sqrt{M}$, which would give a length of $1.7 \mathrm{~mm}$ for the present case. The law by Lasheras et al. (1998) was derived at atmospheric pressure, which could explain the large deviation. In the near-nozzle region $(\mathrm{r}<1 \mathrm{~mm}, \mathrm{z}<5 \mathrm{~mm})$ the liquid phase is densely distributed, and starts to be in a diluted regime at $\mathrm{z} \approx 8 \mathrm{~mm}$. The opening of the spray due to the intermittent flapping of the jet is well visible with the trumpet shape of $\alpha_{L}$ in the region $5<\mathrm{z}<7 \mathrm{~mm}$. Since the flapping does not occur regularly, there is a neat distinction between the region only swept by the flapping, with a volume fraction lower than $10^{-4}$, and the region swept by both the flapping and axial fragmentation $\left(\alpha_{L} \approx 10^{-2}\right)$. The change of opening angle occurring in the outer zone at $7<\mathrm{z}<10 \mathrm{~mm}$ is an artifact due to the entrainment streams.

Downstream of $\mathrm{z}=15 \mathrm{~mm}$, the distribution of the liquid phase is rather homogenous and very diluted with $\alpha_{L} \approx 10^{-4}$. The curved lines of very low volume fraction $\left(\alpha_{L} \approx 10^{-7}\right)$ for $z<5 \mathrm{~mm}$ are due to clusters composed of less than five particles which are torn from the liquid jet and trapped in the recirculation zone. The lines depict the trajectory of these small clusters because of the high sampling frequency. They are not visible in Figs. 19b to 19d because of the filtering of clusters composed of less than five particles.

The map of droplet concentration (Fig. 19b) shows a high concentration directly downstream the nozzle

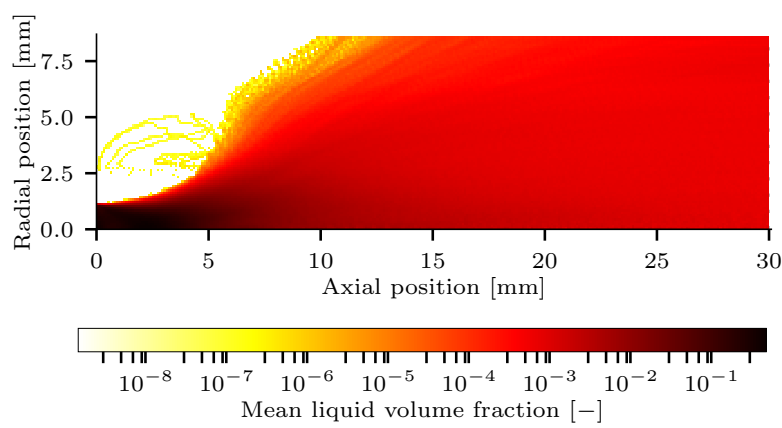

(a)

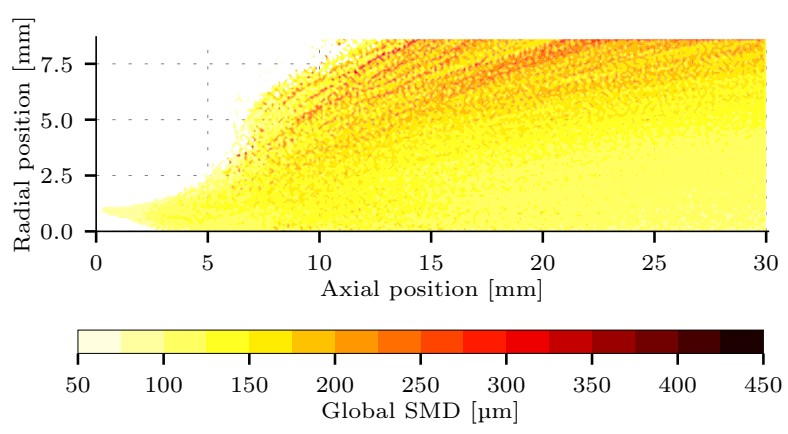

(c)
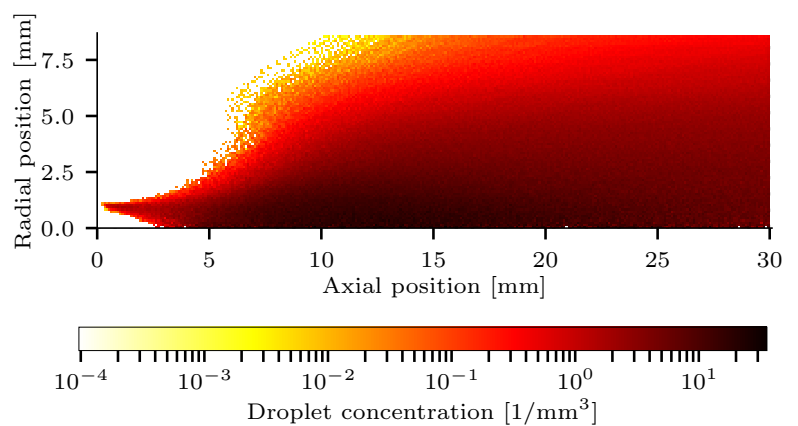

(b)

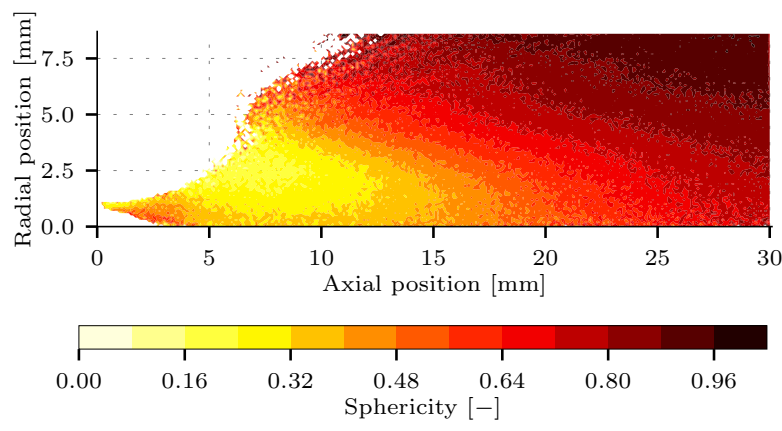

(d)

Figure 19: 2D maps of time average liquid fields on the projection plane.

exit. It corresponds to the liquid lumps that are torn away without undergoing a ligament breakup that would delay the drop formation. This early breakup is not observed in the experiment. Hence, this effect may be due to a too coarse spatial resolution. On this map, the intermittent flapping is also visible with a droplet concentration two orders of magnitude lower at the outer part of the cavity compared to the center region.

On the map of the SMD (Fig. 19c) the effect of oversampling is visible with the trajectories of large particles 
(C)2019. This manuscript version is made available under the CC-BY-NC-ND 4.0 license http://creativecommons.org/licenses/by-nc-nd/4.0/

that generate a dashed line of red dots. This effect is particularly visible in the region only swept by the flapping. This suggest that the flapping mode produces larger droplets than when the jet is axially peeled off. Apart from this, the distribution of the SMD is coherent with the distribution of the liquid fraction and the droplet concentration: the region close to the center line contains more droplets of smaller diameter, whereas the outer region is filled with fewer droplets of larger diameter. Finally, the map of the sphericity (Fig. 19d) brings additional information about the shape of the liquid lumps. At the nozzle exit where small liquid lumps are torn away, the sphericity is high. This means that the droplets are close to spheres and therefore, that the ligaments are very short. The zone of $\varphi<0.32$ for $\mathrm{z}$ between 5 and $10 \mathrm{~mm}$ indicates that the liquid lumps are highly distorted, most likely in the shape of a ligament. For $\mathrm{z}>15 \mathrm{~mm}$, the sphericity increases with increasing $\mathrm{r}$ and $\mathrm{z}$. The zone of constant $\varphi$ could be ellipsoids. Indeed, it is observed that $\partial \varphi / \partial z \sim c_{1} / r$ and $\partial \varphi / \partial r \sim c_{2} / z$, which leads to $\varphi(r, z) \sim c_{1} z^{2}+c_{2} r^{2}$, where $c_{1}$ and $c_{2}$ are constants.

\subsection{Axial evolution of time-averaged liquid quantities}

The 2D fields presented in the previous section can be summed over $r$. The resulting 1D functions only depend on the axial coordinate $z$. They are depicted in Fig. 20. The droplet concentration constantly increases for $z>10 \mathrm{~mm}$, which corroborates the fact that the SMD constantly decreases starting from $\mathrm{z}=10 \mathrm{~mm}$, in virtue of the liquid volume conservation. Note that no secondary droplets were generated when the liquid impacted the outer wall. The liquid was spilled on the wall and sheared towards the outlet by the gas. For $\mathrm{z}>10 \mathrm{~mm}$, the gradient of the SMD is $\approx-1 \mu \mathrm{m} / \mathrm{mm}$. From the point of view of an experimentalist, the axial evolution of the sphericity is interesting because it gives an indication where the droplets are almost spherical, and hence where the PDA technique could be applied successfully. The local maximum at $\mathrm{z} \approx 3 \mathrm{~mm}$ corresponds to the initial droplets torn from the jet, due to a too coarse resolution. Note that smaller droplets are more spherical than large droplets because of the greater influence of the surface tension. Therefore smaller droplets tend to increase the mean sphericity. In particular, droplets of less than 5 particles are dismissed in the post-processing, which leads to an underestimation of the sphericity presented in Figs. 19d and 20. However, since the production of under-resolved droplets is observed roughly everywhere in the numerical domain, the spatial evolution of the sphericity remains unchanged.
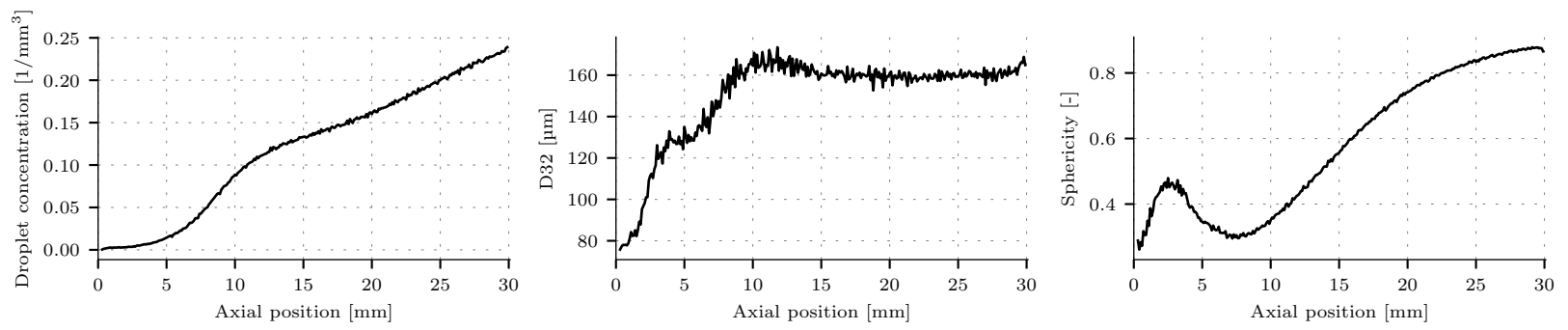

Figure 20: Axial evolution of the number of droplets, SMD and sphericity.

\subsection{Spray characteristics}

Observation of the time series from the simulation revealed large temporal changes of the liquid presence downstream the jet. This is illustrated in Fig. 21 and corresponds to the phenomenon identified by Farago and Chigier (1992) as the super pulsating mode. The presence of the super pulsating mode in this configuration is in agreement with the regime map provided by Farago and Chigier (1992) which depends on the aerodynamic Weber number and the liquid Reynolds number. In order to quantitatively detect this mode, the droplet concentration, the volume fraction and the SMD were monitored versus time at the two different slices depicted in Fig. 12. For each slice, a Fourier Transform was applied to the time signal of the three quantities and the cross spectra were computed. The spectra were improved using windowing and zero padding.

The CSD between the SMD and the droplet concentration signals for the slice at $10<\mathrm{z}<15 \mathrm{~mm}$ is presented in Fig. 22(left), where two peaks are visible at 182 and $880 \mathrm{~Hz}$. For z > $25 \mathrm{~mm}$ (Fig. 22 right), 


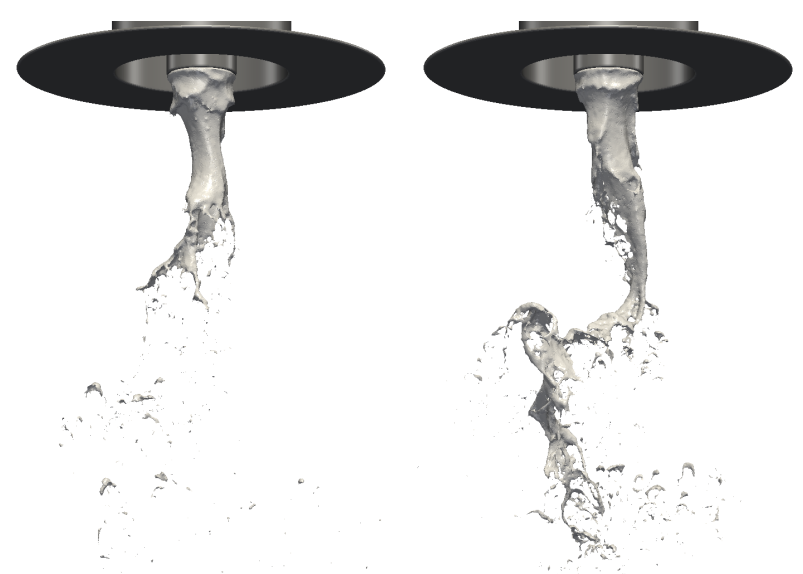

Figure 21: Illustration of the high periodical change between low and high-density regions.

the CSD between the volume fraction and the droplet concentration signals reveals only one peak at $185 \mathrm{~Hz}$ which corroborates the first peak of the first slice. These peaks highlight periodical temporary change in the volume fraction, number density and SMD of the spray, which correspond to the so-called super pulsating mode. To the authors knowledge, this phenomenon was not experimentally quantified previously.
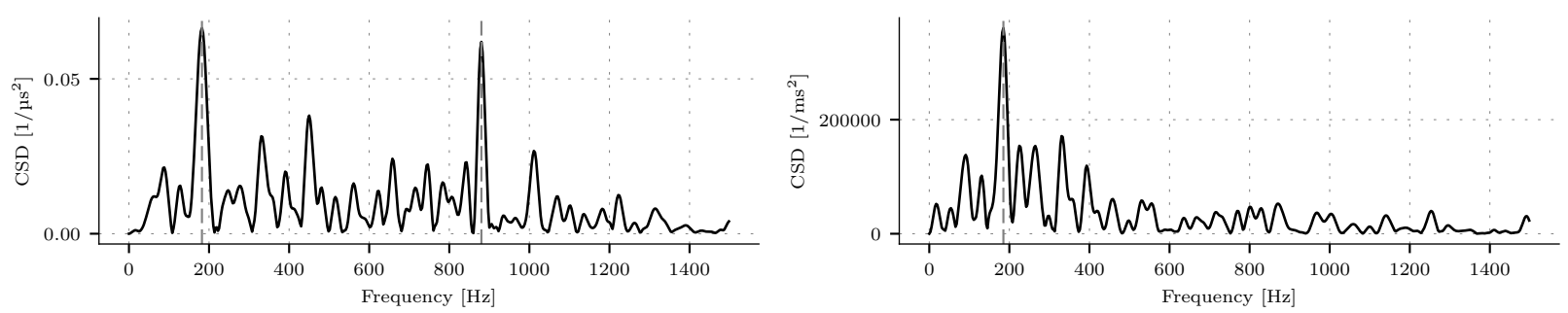

Figure 22: Left: CSD of the SMD and the droplet concentration signals for $10<\mathrm{z}<15 \mathrm{~mm}$. Right: CSD of the volume fraction and the droplet concentration for $\mathrm{z}>25 \mathrm{~mm}$.

The spray characteristics were calculated on the two slices, i.e. (i) for $10<\mathrm{z}<15 \mathrm{~mm}$ in the early stage of the jet breakup and (ii) for $\mathrm{z}>25 \mathrm{~mm}$ at the outlet of the domain. The axial and radial component of the liquid velocity are plotted versus the radial coordinate in Fig. 23. Despite the low velocity of the liquid jet, the maximum axial droplet velocity (Fig. 23 left) is reached at the centerline. This is due to (i) the opening of the turbulent high speed air stream and (ii) the turbulence induced by the local air flow alterations imposed by the flapping liquid jet. In the early stage of the breakup, the maximum axial velocity is already $30 \mathrm{~m} / \mathrm{s}$ and the profile shows different plateaus. At the outlet of the domain, the profile is smoother due to turbulent dispersion of the liquid. The droplets are accelerated to a maximum velocity of $38.6 \mathrm{~m} / \mathrm{s}$ at the center line. The radial velocity of the droplets (Fig. 23 right) is one order of magnitude lower than the axial velocity and decreases at larger $\mathrm{z}$. This is due to the dissipation of the radial momentum that was gained during the initial peel-off of the liquid jet. For the two components $(r, z)$, the velocity is reduced to zero at the outer wall of the domain.

The droplet size volume distribution on the two slices is presented in Fig. 24. Additionally the SMD is marked by the dashed line. The dotted line is a Rosin-Rammler function which best fits the distributions extracted from the simulation. The Rosin-Rammler distribution is one of the typical function to describe the drop size spectrum of a spray generated by this type of nozzle. On slice 1, the density of probability is wider with large liquid lumps up to $1.4 \mathrm{~mm}$, whereas the maximum diameter is circa $0.9 \mathrm{~mm}$ on slice 2 . The SMD decreases from 197 to $137 \mu \mathrm{m}$ between slice 1 and slice 2. It is observed that the shape of the 
(C)2019. This manuscript version is made available under the CC-BY-NC-ND 4.0 license http://creativecommons.org/licenses/by-nc-nd/4.0/
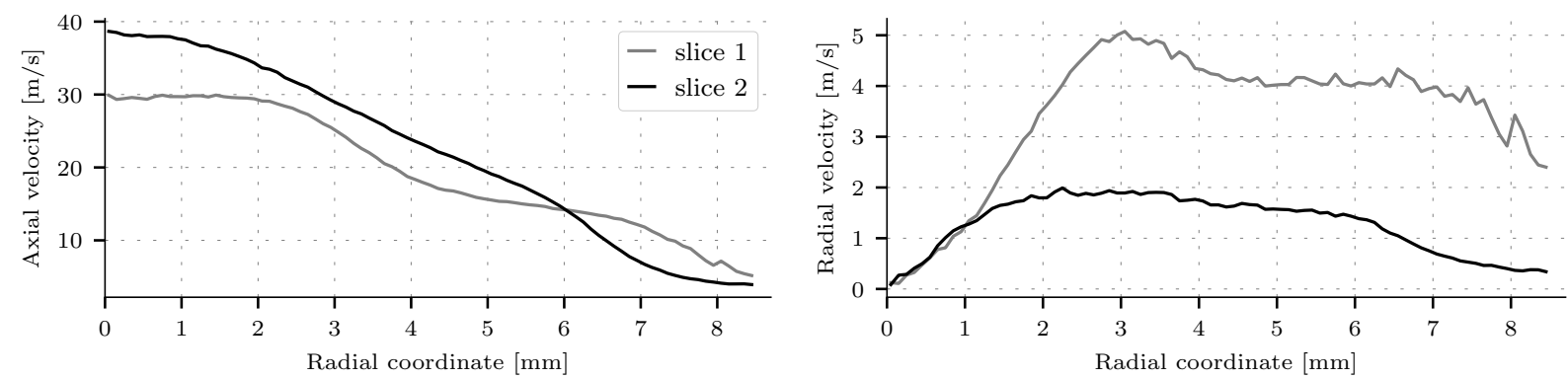

Figure 23: Radial profiles of the liquiq axial (left) and radial (right) velocity.

PDF resolved by the numerical simulation is truncated at the lower diameters range, which compromises the determination of the peak of the PDF. This issue is expected because of the too coarse spatial resolution. Indeed, the small diameters from 0 to $66 \mu \mathrm{m}$ are not captured by the present discretization. Nevertheless, the fitting of the data with the Rosin-Rammler distribution in Fig. 24 shows that the resolved scales of the droplets follow this type of distribution, which is a promising result. It has to be noted that the same configuration was simulated in 2D (Chaussonnet et al., 2018) with a particle size of $20 \mu \mathrm{m}$ and the peak could be resolved. This suggests that in the present 3D case, a slight decrease of the particle size from $33.33 \mu \mathrm{m}$ to $20 \mu \mathrm{m}$ should be enough to capture the peak of the PDF.

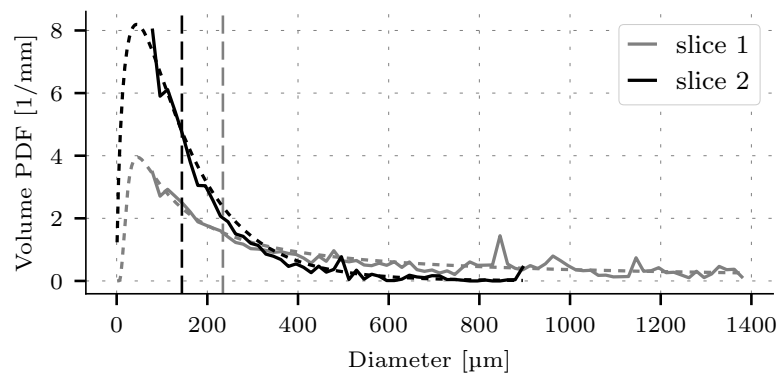

Figure 24: Droplet size volume distribution at the early stage of the breakup (grey solid line) and at the domain outlet (black solid line), and the corresponding Rosin-Rammler distributions (dotted line) which best fit the data from the simulation.

\subsection{Tree of fragmentation}

This part is dedicated to the analysis of the fragmentation tree. Figure 25 shows the axial and radial profiles of the breakup activity $N_{\phi}$ defined in Section 2.4. The axial evolution of $N_{\phi}$ shows that the maximum of the breakup activity occurs at $\mathrm{z} \approx 11 \mathrm{~mm}$, which corresponds to the mean intact length. At the outlet of the domain, $N_{\phi}$ goes to zero, suggesting that the spray is in a stable form, i.e. its drop size distribution will not change anymore. With regards to industrial applications, it is a valuable information to be able to predict were the spray has reached its final drop size distribution. The radial profile of $N_{\phi}$ shows that the most active region is of $0.5<\mathrm{r}<1 \mathrm{~mm}$, which is the position at the prolongation of the liquid duct. It is an unexpected result because one could expect that most of breakup events would occur within the vorticity thickness of the air stream, initially located a $r>1 \mathrm{~mm}$.

Finally, the 2D map of $N_{\phi}$ is depicted in Fig. 26, versus the axial and radial coordinates. It is observed that most of the breakup events occur between $\mathrm{z}=5$ and $15 \mathrm{~mm}$, and for $r$ between 0 and $3 \mathrm{~mm}$. The overall distribution of $N_{\phi}$ seems to be correlated with the distribution of the droplet concentration (Fig. 19b). With regards to industrial application where combustion of the spray is intended, this map could allow to understand where the flame would be stabilized after most of the breakup events. 

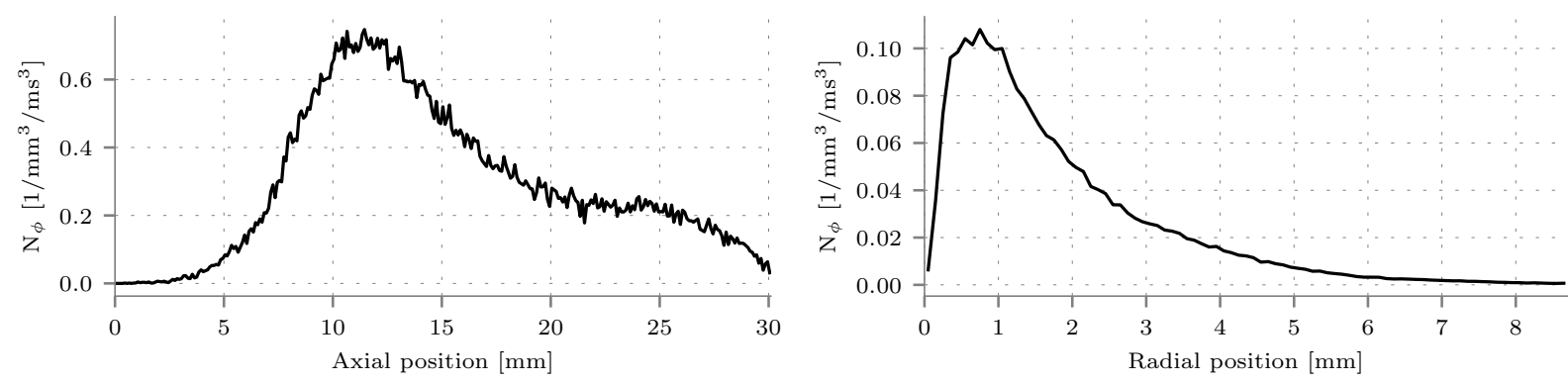

Figure 25: Axial (left) and radial (right) profiles of the number of breakup events per volume and time unit

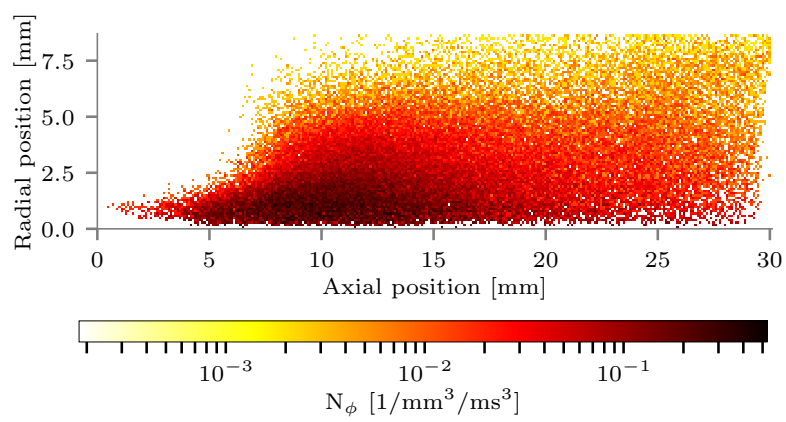

Figure 26: 2D map of the number of breakup events per volume and time unit.

\section{Conclusion}

In this work the air-assisted disintegration of a viscous liquid jet at high pressure was simulated by means of the SPH method, and compared to experimental observations. Qualitatively, the SPH method is able to recover the right type of macroscopic instability that affects the liquid jet. Comparison with high-speed images from the experiment show that the agreement is good both in terms of temporal spatial evolution. The simulation was quantitatively validated in terms of (i) the predicted frequency of the Kelvin-Helmholtz instability and (ii) the distribution of the intact length. Thank to a Eulerian projection, the mean liquid volume fraction, the droplet concentration, the SMD and the droplet sphericity were extracted and timeaveraged. Their 1D profiles and 2D maps are typical for this type of configuration. Moreover, a particular mode of breakup, the super pulsating mode, was observed in the simulation, and its frequency could be estimated for the first time. Concerning the spray characteristics, it was observed that a spatial resolution of $33.33 \mu \mathrm{m}$ is too coarse to resolve the whole drop size PDF. Finally, thanks to unique IDs attributed to the particles, it was possible to monitor the breakup cascade for every individual liquid droplet. This allowed to determine where the spray has reached a steady state regarding the size of the droplets.

In this work the capability of the SPH method to simulate air-assisted atomization was demonstrated. Due to its efficient serial and parallel performance, the computational costs to obtain converged statistics have been substantially reduced. The chain of post-processing tools was applied to obtain the map of different critical spray parameters. It gave the possibility to validate the results of the simulation versus the high-speed images from the experiment. The combination of these characteristics make the SPH method as a suitable candidate for the development of a virtual spray test-rig.

\section{Acknowledgement}

The authors like to thank the Helmholtz Association of German Research Centres (HGF) for funding (Grant No. 34.14.02). This work was performed on the computational resource ForHLR Phase I funded 
(C)2019. This manuscript version is made available under the CC-BY-NC-ND 4.0 license http://creativecommons.org/licenses/by-nc-nd/4.0/

by the Ministry of Science, Research and the Arts Baden-Württemberg and DFG ("Deutsche Forschungsgemeinschaft"). The authors also like to thank Harmut Häfner from KIT-SCC for technical support on the HPC cluster.

Adami, S., Hu, X., and Adams, N. (2010). A new surface-tension formulation for multi-phase SPH using a reproducing divergence approximation. Journal of Computational Physics, 229:5011 - 5021.

Amirshaghaghi, H., Rahimian, M. H., Safari, H., and Krafczyk, M. (2018). Large eddy simulation of liquid sheet breakup using a two-phase lattice boltzmann method. Computers \& Fluids, 160:93-107.

Bonet, J. and Lok, T.-S. L. (1999). Variational and momentum preservation aspects of smooth particle hydrodynamic formulations. Computer methods in applied mechanics and engineering, 180:97 - 115.

Brackbill, J., Kothe, D., and Zemach, C. (1992). A continuum method for modeling surface tension. Journal of Computational Physics, 100:335 - 354.

Braun, S., Koch, R., and Bauer, H.-J. (2016a). Smoothed particle hydrodynamics for numerical predictions of primary atomization. In High Performance Computing in Science and Engineering' 16, pages 321-336. Springer.

Braun, S., Wieth, L., Dauch, T., Keller, M., Chaussonnet, G., Klatt, J.-N., Höfler, C., Koch, R., and Bauer, H.-J. (2016b). HPC predictions of primary atomization with SPH: Challenges and lessons learned. In 11th International SPHERIC Workshop.

Braun, S., Wieth, L., Koch, R., and Bauer, H.-J. (2015a). A framework for permeable boundary conditions in SPH: Inlet, outlet, periodicity. In Proc. of the 10th Internat. SPHERIC Workshop.

Braun, S., Wieth, L., Koch, R., and Bauer, H.-J. (2015b). Influence of trailing edge height on primary atomization: Numerical studies applying the smoothed particle hydrodynamics (SPH) method. In Proceedings of the International Conference on Liquid Atomization and Spray Systems (ICLASS).

Chaussonnet, G., Koch, R., Bauer, H.-J., Sänger, A., Jakobs, T., and Kolb, T. (2018). Smoothed particle hydrodynamics simulation of an air-assisted atomizer operating at high pressure: Influence of non-newtonian effects. Journal of Fluids Engineering, 140(6):061301.

Cleary, P. and Monaghan, J. J. (1999). Conduction modelling using smoothed particle hydrodynamics. Journal of Computational Physics, 148:227 - 264 .

Cleary, P. W. (1998). Modelling confined multi-material heat and mass flows using sph. Applied Mathematical Modelling, 22(12):981-993.

Colagrossi, A. and Landrini, M. (2003). Numerical simulation of interfacial flows by smoothed particle hydrodynamics. Journal of Computational Physics, 191:448 - 475.

Cole, R. H. (1965). Underwater explosions. Dover Publications.

Dauch, T., Braun, S., Wieth, L., Chaussonnet, G., Keller, M., Koch, R., and H.-J., B. (2017). Computational prediction of primary breakup in fuel spray nozzles for aero-engine combustors. In ILASS Europe, 29th Annual Conference on Liquid Atomization and Spray Systems, Valencia, Spain.

Dauch, T., Rapp, T., Chaussonnet, G., Braun, S., Keller, M., Kaden, J., Koch, R., Dachsbacher, C., and Bauer, H.-J. (2018). Highly efficient computation of finite-time lyapunov exponents (ftle) on gpus based on three-dimensional sph datasets. Computers \& Fluids, 175:129 - 141.

Dhivyaraja, K., Gaddes, D., Freeman, E., Tadigadapa, S., and Panchagnula, M. (2019). Dynamical similarity and universality of drop size and velocity spectra in sprays. Journal of Fluid Mechanics, 860:510-543.

Dumouchel, C. (2008). On the experimental investigation on primary atomization of liquid streams. Experiments in Fluids, 45:371-422.

Edelsbrunner, H., Kirkpatrick, D., and Seidel, R. (1983). On the shape of a set of points in the plane. IEEE Transactions on information theory, 29(4):551-559.

Español, P. and Revenga, M. (2003). Smoothed dissipative particle dynamics. Phys. Rev. E, 67:026705.

Farago, Z. and Chigier, N. (1992). Morphological classification of disintegration of round liquid jets in a coaxial air stream. Atomization and Sprays, 2:137-153.

Gingold, R. and Monaghan, J. J. (1977). Smoothed particle hydrodynamics-theory and application to non-spherical stars. Mon. Not. R. Astron. Soc., 181:375-389.

Gorokhovski, M. and Saveliev, V. (2008). Statistical universalities in fragmentation under scaling symmetry with a constant frequency of fragmentation. Journal of Physics D: Applied Physics, 41(8):085405.

Höfler, C., Braun, S., Koch, R., and Bauer, H.-J. (2011). Towards the numerical prediction of primary atomization using smoothed particle hydrodynamics. In Proceedings of the European Conference on Liquid Atomization and Spray Systems (ILASS).

Hu, X. and Adams, N. (2006). A multi-phase SPH method for macroscopic and mesoscopic flows. Journal of Computational Physics, 213:844-861.

Hussein, H. J., Capp, S. P., and George, W. K. (1994). Velocity measurements in a high-Reynolds-number, momentumconserving, axisymmetric, turbulent jet. Journal of Fluid Mechanics, 258:31-75.

Jakobs, T., Djordjevic, N., Sanger, A., Zarzalis, N., and Kolb, T. (2015). Influence of reactor pressure on twin-fluid atomization: basic investigations on burner design for high-pressure entrained flow gasifier. Atomization and Sprays, 25(12).

Kapur, J. N., Sahoo, P. K., and Wong, A. K. (1985). A new method for gray-level picture thresholding using the entropy of the histogram. Computer vision, graphics, and image processing, 29(3):273-285.

Lasheras, J., Villermaux, E., and Hopfinger, E. J. (1998). Break-up and atomization of a round water jet by a high-speed annular air jet. Journal of Fluid Mechanics, 357:351-379.

$\mathrm{Li}, \mathrm{X}$. and Soteriou, M. C. (2018). Detailed numerical simulation of liquid jet atomization in crossflow of increasing density. International Journal of Multiphase Flow. 
(c)2019. This manuscript version is made available under the CC-BY-NC-ND 4.0 license http://creativecommons.org/licenses/by-nc-nd/4.0/

Maciá, F., Antuono, M., González, L. M., and Colagrossi, A. (2011). Theoretical analysis of the no-slip boundary condition enforcement in SPH methods. Progress of theoretical physics, 125(6):1091-1121.

Matas, J.-P., Delon, A., and Cartellier, A. (2018). Shear instability of an axisymmetric air-water coaxial jet. Journal of Fluid Mechanics, 843:575-600.

Monaghan, J. J. (1994). Simulating free surface flows with SPH. J. Comput. Phys., 110(2):399-406.

Monaghan, J. J. (2005). Smoothed particle hydrodynamics. Reports on Progress in Physics, 68:1703-1759.

Monaghan, J. J. and Kocharyan, A. (1995). SPH simulation of multi-phase flow. Computer Physics Communications, 87:225 -235 .

Müller, T., Sänger, A., Habisreuther, P., Jakobs, T., Trimis, D., Kolb, T., and Zarzalis, N. (2016). Simulation of the primary breakup of a high-viscosity liquid jet by a coaxial annular gas flow. International Journal of Multiphase Flow, 87:212 - 228.

Pereira, G. G., Cleary, P. W., and Serizawa, Y. (2018). Prediction of fluid flow through and jet formation from a high pressure nozzle using smoothed particle hydrodynamics. Chemical Engineering Science, 178:12-26.

Rivest, R. (1991). The md5 message-digest algorithm. https://tools.ietf .org/html/rfc1321. [Online; accessed 27-March2018].

Rosenfeld, A. and Pfaltz, J. L. (1966). Sequential operations in digital picture processing. Journal of the ACM (JACM), 13(4):471-494.

Salvador, F., Ruiz, S., Crialesi-Esposito, M., and Blanquer, I. (2018). Analysis on the effects of turbulent inflow conditions on spray primary atomization in the near-field by direct numerical simulation. International Journal of Multiphase Flow.

Sänger, A., Jakobs, T., Djordjevic, N., and Kolb, T. (2014). Effect of primary instability of a high viscous liquid jet on the spray quality generated by a twin-fluid atomizer. In Proceedings of the European Conference for Liquid Atomization and Spray Systems (ILASS).

Sänger, A., Jakobs, T., Djordjevic, N., and Kolb, T. (2015). Experimental investigation on the influence of ambient pressure on twin-fluid atomization ofliquids with various viscosities. In Proceedings of the Triennal International Conference on Liquid Atomization and Spray System (ILASS).

Shao, C., Luo, K., Yang, Y., and Fan, J. (2017). Detailed numerical simulation of swirling primary atomization using a mass conservative level set method. International Journal of Multiphase Flow, 89:57-68.

Sun, P., Colagrossi, A., Marrone, S., and Zhang, A. (2016). Detection of lagrangian coherent structures in the sph framework. Computer Methods in Applied Mechanics and Engineering, 305:849-868.

Szewc, K., Pozorski, J., and Minier, J.-P. (2012). Analysis of the incompressibility constraint in the smoothed particle hydrodynamics method. International Journal for Numerical Methods in Engineering, 92(4):343-369.

Takeda, H., Miyama, S., and Sekiya, M. (1994). Numerical simulation of viscous flow by smoothed particle hydrodynamics. Progress of Theoretical Physics, 92(5):939 - 960.

Warncke, K., Gepperth, S., Sauer, B., Sadiki, A., Janicka, J., Koch, R., and Bauer, H.-J. (2017). Experimental and numerical investigation of the primary breakup of an airblasted liquid sheet. International Journal of Multiphase Flow, 91:208-224. 